\title{
PATH DERIVATIVES: A UNIFIED VIEW OF CERTAIN GENERALIZED DERIVATIVES
}

BY

\author{
A. M. BRUCKNER ${ }^{1}$, R. J. O'MALLEY ${ }^{1}$ AND B. S. THOMSON ${ }^{2}$
}

\begin{abstract}
A collection $E=\left\{E_{x}: x \in R\right\}$ is a system of paths if each set $E_{x}$ has $x$ as a point of accumulation. For such a system $E$ the derivative $F_{l}^{\prime}(x)$ of a function $F$ at a point $x$ is just the usual derivative at $x$ relative to the set $E_{\mathrm{x}}$. The goal of this paper is the investigation of properties that $F$ and its derivative $F_{l}^{\prime}$ : must have under certain natural assumptions about the collection $E$. In particular, it is shown that most of the familiar properties of approximate derivatives and approximately differentiable functions follow in this setting from three conditions on the collection $E$ relating to the "thickness" of the sets $E_{\mathrm{x}}$ and the way in which the sets intersect.
\end{abstract}

1. Introduction. In this paper we introduce the concept of a path derivative as a unifying approach to the study of a number of generalized derivatives. There have been, in recent years, numerous articles showing that certain generalized derivatives, which can often substitute for the ordinary derivative when the latter is not known to exist, share many properties of the ordinary derivative. For example, an approximate derivative has the Darboux property, is in the first class of Baire, possesses the Denjoy property and is in Zahorski's classes $\Re_{2}$ and $\Re_{3}$ and Weil's class $\mathscr{Z}$. Each approximate derivative $f$ can be represented in the form $f=g^{\prime}+h k^{\prime}$ where $g, h$, and $k$ are differentiable; the restriction of such a function $f$ to a nowhere dense set can be extended so as to be a derivative on all of $R$. Furthermore, an approximately differentiable function $F$ shares many properties of ordinary primitives: it is of generalized absolute continuity $[A C G]$, is differentiable on a dense open set, has the Darboux property, assumes local extrema, and is determined by its values on any dense set. Finally any monotonicity theorem that is valid for differentiable functions has an analogue for approximately differentiable functions. For precise formulations and proofs of these remarks see $[1,4,10,16,18,26,30,37$, 41, 42, and 43].

Certain other derivatives also possess some of these properties. The Peano derivative shares most of these (see $[23,30,40-42])$, while the preponderant $[3,9$ and 10], the selective $[19,25,27$, and 28] and the sequential [32] possess only a few. The Dini derivatives, on the other hand, may have none of these properties.

\footnotetext{
Received by the editors January 20, 1982 and, in revised form, February 9, 1983.

1980 Mathematics Subject Classification. Primary 26A24; Secondary 26A21.

Key words and phrases. Derivatives, approximate derivatives, Peano derivatives, functions of Baire class 1, Darboux functions.

'The work of these authors was supported in part by a grant from the National Science Foundation.

${ }^{2}$ The work of this author was supported in part by a grant from the Natural Sciences and Engineering Research Council of Canada.
} 
It is natural, then, to ask for a framework within which all of these derivatives can be expressed and which reveals how the various properties arise. The perspective we choose is to consider just those derivatives for which the derivative of a function $F$ at a point $x$ can be viewed as

$$
\lim _{\substack{y \rightarrow x \\ y \in E_{\text {, }}}} \frac{F(y)-F(x)}{y-x}
$$

for appropriate choices of sets $E_{x}$. One generalized derivative, then, differs from another only in the choice of the family of sets $\left\{E_{x}: x \in R\right\}$ through which the difference quotient passes to its limit. For example, an approximately differentiable function $F$ permits a choice of sets $\left\{E_{x}: x \in R\right\}$ so that each $E_{x}$ has density 1 at $x$; for a Dini derivative the sets may only consist of sequences converging to $x$. Although such a framework will exclude from consideration symmetric derivatives, it will, nonetheless, include any generalized derivative for which the derivative at a point is a derived number of the function at that point.

We shall see that much of the information concerning the behaviour of a generalized derivative is contained in the geometry of the collection $E=\left\{E_{x}\right.$ : $x \in R\}$. In particular, it happens that a generalized derivative that permits a choice of sets $E_{x}$, meeting only three conditions, must have most of the properties stated above for approximate derivatives. These conditions relate to the "thickness" of the $E_{x}$ and the way in which the sets intersect.

We begin with a preliminary section giving the basic definitions for our structure and a few elementary observations about that structure. In $\S 3$ we present the basic conditions on that structure which yield our results. $\$ 4$ contains some technical lemmas on extreme derivates. The remainder of the paper is concerned with generalized derivatives and generalized primitives. The properties of the primitives are explored in $\$ 5$ and the properties of exact generalized derivatives in $\S 6$. Frequently differentiability in a generalized sense entails ordinary differentiability on certain sets; this phenomenon is studied in $§ 7$. Finally, some diverse notions appear in $\S 8$. Although there are a number of interesting, unanswered questions that we have not addressed here, this paper contains the essential theory for these path derivatives.

2. Preliminaries. The fundamental objects of our study are systems of paths on the real line.

2.1 Definition. Let $x \in R$. A path leading to $x$ is a set $E_{x} \subset R$ such that $x \in E_{x}$ and $x$ is a point of accumulation of $E_{x}$. A system of paths is a collection $E=\left\{E_{x}\right.$ : $x \in R\}$ such that each $E_{x}$ is a path leading to $x$.

2.2 Definition. Let $F: R \rightarrow R$ and let $E=\left\{E_{x}: x \in R\right\}$ be a system of paths. If

$$
\lim _{\substack{y \rightarrow x \\ y \in E_{x}}} \frac{F(y)-F(x)}{y-x}=f(x)
$$

is finite then we say that $F$ is $E$-differentiable at $x$ and write $F_{E}^{\prime}(x)=f(x)$. If $F$ is $E$-differentiable at every point $x$ then we say simply that $F$ is $E$-differentiable; we call $F$ an $E$-primitive and $f$ an $E$-derivative. 
We shall speak loosely of such objects as path derivatives. Note that any function $F$ differentiable in the ordinary sense at a point $x$ is necessarily $E$-differentiable at $x$ for any choice of paths $E$ and that $F_{E}^{\prime}(x)=F^{\prime}(x)$; also whenever $F_{I:}^{\prime}(x)$ exists it is a finite derived number of the function $F$ at the point $x$. Of course the converse is also true: if at every point $x$ a function $F$ has at least one finite derived number $f(x)$, then a choice of paths $E=\left\{E_{x}: x \in R\right\}$ can be made so that $F_{E}^{\prime}(x)=f(x)$ everywhere.

This allows, then, the expression of any generalized derivative as a path derivative provided only that, at each point, that generalized derivative is a derived number of the function. As a great many theories have this property our language will include many of the standard generalized derivatives. However, it is not merely the choice of paths $E=\left\{E_{x}: x \in R\right\}$ that is our concern, but the choice of quite substantial paths. In order to provide some language with which to express these choices of paths we introduce a classification of paths within which various generalized derivatives can be expressed.

2.3 Definition. We shall classify systems of paths according to the following scheme. A system of paths $E=\left\{E_{x}: x \in R\right\}$ will be said to be

(2.3.1) of ordinary type if each $E_{x}$ is a neighborhood of $x$;

(2.3.2) of $(1,1)$-density type if each $E_{x}$ has density 1 at $x$;

(2.3.3) of $(\rho, \lambda)$-density type if each $E_{x}$ has right lower density exceeding $\rho$ and left lower density exceeding $\lambda$ at $x$ (if $\rho=1$ or $\lambda=1$ then the corresponding density is to be equal to 1$)$;

(2.3.4) of congruent type if $E_{x}=Q+x$ for each $x$ where $Q$ is a set having 0 as a member and as a point of accumulation;

(2.3.5) of sequential-congruent type if $E_{x}=Q+x$ for each $x$ where $Q=$ $\left\{0, h_{1}, h_{2}, h_{3}, \ldots\right\}$ and $\left\{h_{n}\right\}$ is a fixed sequence convergent to 0 ;

(2.3.6) of qualitative type if each $E_{x}$ is residual in a neighborhood of $x$;

(2.3.7) of unilateral type if each $E_{x}$ is a one-sided neighborhood of $X$ from one invariable side; and

(2.3.8) of $(\rho,-)$-density type if each $E_{x}$ has right lower density exceeding $\rho$ (or equal to 1 if $\rho=1)$. (-, $\lambda)$-density type is similarly defined.

A number of familiar derivatives can be expressed as path derivatives along systems of paths of these types. Certainly an ordinary derivative is just a path derivative for a system $E$ that is of ordinary type. For an approximate derivative there is a system $E$ of $(1,1)$-density type and for a preponderant derivative a system of $(1 / 2,1 / 2)$-density type. The congruent derivative [35] is just a path derivative for a path system of congruent type, and similarly the sequential derivative [32] is again such a path derivative for an $E$ of sequential-congruent type. The qualitative derivative corresponds to systems of qualitative type [20]. One-sided derivatives obviously permit unilateral paths and, finally, a one-sided approximate derivative corresponds to a choice of paths of the type $(1,-)$ density or $(-, 1)$ density.

2.4 RemarK. A more general, if somewhat less revealing, approach to our investigations is to introduce the notion of a "filter derivative." For each $x \in R$ let there be given a filter $N(x)$ converging to $x$. Then $F_{N}^{\prime}(x)=f(x)$ exists for a function 
$F$ if for every $\varepsilon>0$ there is an $\eta \in N(x)$ with

$$
\left|\frac{F(y)-F(x)}{y-x}-f(x)\right|<\varepsilon
$$

for every $y \in \eta, y \neq x$. For example, if $N(x)$ consists of all sets having density 1 at $x$ this type of derivative will characterize approximate derivatives. This has the advantage of defining a structure that can be used to study a generalized derivative for whole classes of functions; path derivatives, on the other hand, require choices of paths dependent on the function under study.

Nevertheless, our approach allows us considerable flexibility. For example, one often encounters a situation in which a function has a generalized derivative a.e. with some further knowledge available about the derived numbers on the exceptional measure zero set. A path approach may be natural to apply in this situation, whereas a filter derivative might be awkward. Also by dealing always with a specific system of paths tailored to the function, one can make alterations in the paths appropriate to the study of that function: if $F$ is continuous and $E$-differentiable then the paths can be altered in a number of interesting ways. One can replace $E$ by the system $E_{1}=\left\{\bar{E}_{x}: x \in R\right\}$, or one can find open sets $G_{x} \supset E_{x} \backslash\{x\}$ so that $F$ remains differentiable for the system $\left\{G_{x} \cup\{x\}: x \in R\right\}$.

3. The basic conditions. The facts that a function $F$ is an $E$-primitive and that $f(x)=F_{E}^{\prime}(x)$, for a system of paths $E=\left\{E_{x}: x \in R\right\}$, say nothing about the two functions $F$ and $f$ beyond the obvious fact that at each point $x$ the number $f(x)$ is a derived number of the function $F$. By a theorem of Jarnik [17], we know there are continuous functions $F$ that have, at every point, each real number as a derived number; consequently, any function $f$ can be realized as a path derivative of such a function $F$. For future reference we state this as a theorem.

3.1 THEOREM. There is a continuous function $F$ such that, given any function $f$ on $R$, a system of paths $E=\left\{E_{x}: x \in R\right\}$ can be found so that $F_{E}^{\prime}=f$.

A similar assertion is available for systems $E$ that are of sequential-congruent type (see the discussion in [4, pp. 115-117]). By using a theorem of Scholz [34], one can require in this theorem that, for almost every $x$, the sets $E_{x}$ have upper density 1 . Thus, even though most of the sets $E_{x}$ are in some sense "thick", nothing can be said. Our first objective is to define useful conditions on the sets $\left\{E_{x}\right\}$ so that various inferences can be drawn.

We begin with properties that are strictly local in character.

3.2 Definition. Let $E=\left\{E_{x}: x \in R\right\}$ be a system of paths. (If $E$ has any of these properties at each point, then we say that $E$ has that property.)

(3.2.1) $E$ is said to be bilateral at $x$ if $x$ is a bilateral point of accumulation of $E_{x}$.

(3.2.2) $E$ is said to be nonporous from the left (right) at $x$ if $E_{x}$ has left (right) porosity 0 at $x$.

(3.2.3) $E$ is said to be nonporous at $x$ if $E_{x}$ has porosity 0 at $x$.

The basic definition of porosity $[2, \mathbf{1 1}, 13]$ of a set $E$ at $x$ from the right is the value $\lim \sup _{r \rightarrow 0^{+}} l(x, r, E) / r$, where $l(x, r, E)$ denotes the length of the largest open 
interval contained in the set $(x, x+r) \cap(R \backslash E)$. (Porosity 0 at $x$ means both right and left porosity 0 . Note that a nonporous system is necessarily bilateral.)

Each of these properties is local, and, alone, none of them has any global implications. Global properties of derivatives appear to arise from the fact that many of the sets $\left\{E_{x}: x \in R\right\}$ must intersect in some fashion. Below we list a variety of intersection properties for paths. The first of these is the most important and plays a central role in the subsequent theory; the remaining properties have much more limited application.

3.3 Definition. Let $E=\left\{E_{x}: x \in R\right\}$ be a system of paths. $E$ will be said to satisfy the condition listed below if there is associated with $E$ a positive function $\delta$ on $R$ so that whenever $0<y-x<\min \{\delta(x), \delta(y)\}$, the sets $E_{x}$ and $E_{y}$ intersect in the stated fashion:

(3.3.1) intersection condition (I.C.): $E_{x} \cap E_{y} \cap[x, y] \neq \varnothing$;

(3.3.2) internal intersection condition (I.I.C.): $E_{x} \cap E_{y} \cap(x, y) \neq \varnothing$;

(3.3.3) external intersection condition (E.I.C.):

$$
E_{x} \cap E_{y} \cap(y, 2 y-x) \neq \varnothing \quad \text { and } \quad E_{x} \cap E_{y} \cap(2 x-y, x) \neq \varnothing ;
$$

(3.3.4) external intersection condition, parameter $m$ (E.I.C. $[m]$ )

$$
E_{x} \cap E_{y} \cap(y,(m+1) y-m x) \neq \varnothing, \quad E_{x} \cap E_{y} \cap((m+1) x-m y, x) \neq \varnothing ;
$$

(3.3.5) one-sided external intersection condition, parameter $m$ : as for (3.3.3) and (3.3.4), but only one of the intersections need be nonempty.

We pause to prove a result which is closely related to the motivation for focusing on these intersection properties. The notion of a selective derivative was introduced in [25], and a number of derivatives (approximate, preponderant, qualitative) were shown to be expressible as selective derivatives. Any function of two variables $p(x, y)$ satisfying $p(x, y)=p(y, x)$, and $p(x, y)$ is between $x$ and $y$ for all $x \neq y$, is called a selection. For a fixed function $F$, selection $p$, and point $x_{0}$, the selective derivative $F_{p}^{\prime}\left(x_{0}\right)$ is, simply,

$$
\lim _{y \rightarrow x_{0}} \frac{F\left(p\left(x_{0}, y\right)\right)-F\left(x_{0}\right)}{p\left(x_{0}, y\right)-x_{0}} .
$$

Here, we prove that any path derivative, for paths that are bilateral and satisfy the I.I.C., is expressible as a selective derivative; this can be considered a generalization of Theorem 16 of [25]. The converse proposition, that every selective derivative can be realized as a path derivative relative to such a system, is an open question.

3.4 TheOREM. Let $E=\left\{E_{x}: x \in R\right\}$ be a system of paths that is bilateral and satisfies the I.I.C. Then there is a selection $p$ such that every E-differentiable function $F$ is selectively differentiable relative to $p$, and $F_{p}^{\prime}(x)=F_{E}^{\prime}(x)$ for all $x$.

Proof. Let $\delta$ be the function associated with $E$ for the I.I.C. We define $p(x, y)$ for $x<y$ (if $y<x$, let $p(x, y)=p(y, x))$ as follows:

(i) if $0<y-x<\min \{\delta(x), \delta(y)\}$, take any $p(x, y)$ in $E_{x} \cap E_{y} \cap(x, y)$;

(ii) if $0<y-x<\delta(x)$, but $y-x \geqslant \delta(y)$, then take $p(x, y) \in E_{x} \cap(x, y)$; 
(iii) if $0<y-x<\delta(y)$, but $y-x \geqslant \delta(x)$, then take $p(x, y) \in E_{y} \cap(x, y)$; and finally

(iv) if $y-x \geqslant \delta(x)$, and $y-x \geqslant \delta(y)$, take $p(x, y)=(x+y) / 2$. Now if $F_{E}^{\prime}(x)$ exists, it is routine to check that

$$
\lim _{h \rightarrow 0} \frac{F(p(x, x+h))-F(x)}{p(x, x+h)-x}=F_{E}^{\prime}(x),
$$

and the theorem is proved. (Here, as in [25], we intend $p(x, x+h)$, for $h<0$, to signify $p(x+h, x)$.)

3.5 THEOREM. The chart indicates the conditions satisfied, in general, by systems of paths of the type listed in the first column.

\begin{tabular}{|l|l|l|l|l|l|l|l|} 
type & bilateral & nonporous & I.C. & I.I.C. & E.I.C. & $\begin{array}{l}\text { E.I.C. } \\
{[\mathrm{m}]}\end{array}$ & $\begin{array}{l}\text { one-sided } \\
\text { E.I.C. }[\mathrm{m}]\end{array}$ \\
\hline \hline ordinary & yes & yes & yes & yes & yes & yes & yes \\
\hline$(1,1)$ density & yes & yes & yes & yes & yes & yes & yes \\
\hline $\begin{array}{l}(\rho, \lambda) \text { density, } \\
\rho+\lambda \geqslant 1\end{array}$ & yes & no & yes & yes & no & no & no \\
\hline$\left(\frac{3}{4}, \frac{3}{4}\right)$ density & yes & no & yes & yes & yes & yes & yes \\
\hline $\begin{array}{l}(\rho, \lambda) \text { density, } \\
\rho>\frac{1}{2} \text { and } \lambda>\frac{1}{2}\end{array}$ & yes & no & yes & yes & no & yes & yes \\
\hline qualitative & yes & yes & yes & yes & yes & yes & yes \\
\hline unilateral & no & no & yes & no & no & no & yes \\
\hline$(\rho,-)$ density, $\rho>\frac{1}{2}$ & no & no & no & no & no & no & yes \\
\hline
\end{tabular}

Proof. For the most part the verifications are either elementary or straightforward. For example, it is easy to see that a set $E_{x}$ that has density 1 at $X$ must have zero porosity at that point, and a set having density less than 1 may well be porous.

If $E$ is of type $(\rho, \lambda)$ density with $\rho+\lambda \geqslant 1$, we choose the function $\delta$ so that

$$
\left|E_{x} \cap(x, x+t)\right|>\rho t \text { and }\left|E_{x} \cap(x-t, x)\right|>\lambda t
$$

whenever $0<t<\delta(x)$. Then, if $0<y-x<\min \{\delta(x), \delta(y)\}$, we have $\mid E_{x} \cap$ $(x, y) \mid>\rho(y-x)$ and $\left|E_{y} \cap(x, y)\right|>\lambda(y-x)$. Since $\rho+\lambda \geqslant 1$, these two sets must have a point in common, which proves both I.C. and I.I.C.

Let $E$ be of type $\left(\frac{3}{4},-\right)$ density. Choose $\delta$ so that $\left|E_{x} \cap(x, x+t)\right|>3 t / 4$ if $0<t<2 \delta(x)$. Then, if $0<y-x<\min \{\delta(x), \delta(y)\}$, we must have

$$
\left|E_{x} \cap(x, x+2(y-x))\right|>3(2(y-x)) / 4=3(y-x) / 2,
$$

and hence

$$
\left|E_{x} \cap(y, 2 y-x)\right|>(y-x) / 2 .
$$

Since $\left|E_{y} \cap(y, y+(y-x))\right| \geqslant 3(y-x) / 4$, it is clear that there must be a point in the intersection $E_{x} \cap E_{y} \cap(y, 2 y-x)$. This establishes the one-sided E.I.C. for 
such an $E$. Similarly, one may show that if $E$ is of type $\left(\frac{3}{4}, \frac{3}{4}\right)$ density, then $E$ satisfies E.I.C. For density $\rho$ and $\lambda$, with $\frac{1}{2}<\rho<\frac{3}{4}$, and $\frac{1}{2}<\lambda<\frac{3}{4}$, the arguments can be modified to show that E.I.C. $[m]$ holds for an appropriate choice of parameter $m$.

Systems of type $\left(\frac{1}{2}, \frac{1}{2}\right)$ density do not satisfy, in general, an E.I.C. $[m]$; but all our theorems can be extended to them by decomposing the line into a sequence of sets $\left\{X_{n}\right\}_{n=3}^{\infty}$ so that, for $x \in X_{n}$, the density of $E_{x}$ at $x$ exceeds $(n+2) / 2 n$, and then the E.I.C. can be used to yield results on each set of the sequence. Thus, those theorems that use the E.I.C. apply to preponderant derivatives, but vith some technical modifications; we shall not carry out this in detail but merely mention, where it is appropriate, that such a theorem is applicable to the preponderant derivative.

Theorem 3.5 concerns itself with a catalogue of the various familiar types of paths that possess one or more of our properties. For the most part, the paths here involve density considerations that immediately supply the needed intersection condition or nonporosity property. It is less obvious that other types of derivatives will also permit an expression as path derivatives along systems of paths having desirable properties. In our next theorem, we prove that an exact Peano derivative can be expressed as a path derivative of a function along a system of paths that are nonporous. Whether these paths can even be chosen so as to satisfy any of our intersection conditions is an open question. For definitions of the $k$ th Peano derivative, $F^{(k)}$, of a function $F$ see $[23,30,40$ or 42$]$. Our theorem is really only an interpretation in our language of the observation in [30, Lemma 3.5] that the $k$ th Peano derivative $F^{(k)}(x)$ at a point $x$ must be a derived number of the $(k-1)$ st Peano derivative $F^{(k-1)}$.

3.6 THEOREM. Let $F$ be a function having everywhere jth Peano derivatives $F^{(j)}$ of orders $j=1,2,3, \ldots, k$. Then there is a nonporous system of paths $E=\left\{E_{x}: x \in R\right\}$ for which $F_{E}^{(k-1) '}(x)=F^{(k)}(x)$ holds at all $x$.

Proof. The expression of $F^{(k)}$ as a path derivative of $F^{(k-1)}$ is already given by [30, Lemma 3.5] (see also [41, p. 371]), so we need only show that the conditions in that lemma supply, in fact, a nonporous system of paths. The proof can be reduced then to showing the validity of the following lemma.

(3.6.1) Let $F$ be a function that has the following property at a point $x$ : there is $a$ number $\lambda$ so that for every $\varepsilon>0$ there is a $\delta=\delta(\varepsilon)>0$ such that, whenever $0<y-x<\delta$, there must be a number $z$ with $0<y-z<\varepsilon(y-x)$ and $|F(z)-\lambda|$ $<\varepsilon$. Then there is a path $E_{x}$ leading to $x$ and nonporous on the right at $x$ such that

$$
\lim _{\substack{y \rightarrow x \\ y \in E_{x}}} F(y)=\lambda
$$

To prove (3.6.1) let us define a sequence of numbers $\delta_{k}$ so that $0<\delta_{k} \leqslant \delta(1 / k)$ and $\delta_{k+1}<\delta_{k} / 2$, and define the set $E_{x}$ as

$$
E_{x}=x+\bigcup_{k=1}^{\infty}\left[\delta_{k+2}, \delta_{k}\right) \cap\{z:|F(z)-\lambda|<1 / k\} .
$$


It is certainly true that $\lim _{y \rightarrow x, y \in E_{1}} F(y)=\lambda$ (although this is vacuous if $x$ is not a point of accumulation of $E_{x}$ ), so the lemma is proved provided only that we can show $E_{x}$ is nonporous on the right at $x$.

Suppose not. Then there must exist a number $\frac{1}{2}<\theta<1$ and a sequence of numbers $h_{k} \downarrow 0$ with $\left(x+\theta h_{k}, x+h_{k}\right) \cap E_{x}=\varnothing$ for every index $k$. Choose an integer $k_{0}$ larger than $(1-\theta)^{-1}$ (i.e. so that if $k \geqslant k_{0}$, then $1-1 / k>\theta$ ) and let $j_{0}$ be the first index for which $h_{j_{0}}<\delta_{k_{0}}$. Fix $k$ so that $\delta_{k+1} \leqslant h_{j_{0}}<\delta_{k}$, and note that $k \geqslant k_{0}$.

Since $h_{j_{0}}<\delta_{k} \leqslant \delta(1 / k)$, there must be, by the assumptions of the lemma, a point $z$ with $x<z<x+h_{j_{0}},\left(x+h_{j_{0}}\right)-z<\left(x+h_{j_{0}}-x\right) / k=h_{j_{0}} / k$ and $|F(z)-\lambda|<$ $1 / k$.

We then have the inequalities,

$$
\begin{aligned}
x+\delta_{k+2} & <x+\frac{1}{2} \delta_{k+1}<x+\theta \delta_{k+1} \leqslant x+\theta h_{j_{0}} \\
& <x+\left(1-\frac{1}{k}\right) h_{j_{0}}<z<x+h_{j_{0}}<\delta_{k} .
\end{aligned}
$$

From this, then, we see that $|F(z)-\lambda|<1 / k$ and $\delta_{k+2} \leqslant z<\delta_{k}$, so $z \in E_{x}$; but also $x+\theta h_{j_{0}}<z<x+h_{j_{0}}$, so $z \in\left(x+\theta h_{j_{0}}, x+h_{j_{0}}\right)$. This contradicts the fact that $E_{x} \cap\left(x+\theta h_{j}, x+h_{j}\right)=\varnothing$ for all $j$, and the lemma is proved.

The theorem follows directly now from [30, Lemma 3.5] and (3.6.1), together with a left-hand version of (3.6.1).

We conclude this section by systematizing some technical manipulations that will appear nearly every time one of these intersection conditions is invoked. One of the most frequently used tools of differentiation theory involves a decomposition of a set into a sequence of sets, each of which has some kind of uniform behaviour for the derivative in question. While this device is almost universally used in the literature, it appears that it has never been formalized or given a name. We shall refer to this as a $\delta$-decomposition.

3.7 Definition. Let $\delta$ be a positive function and let $X$ be a set of real numbers. By a $\delta$-decomposition of $X$ we shall mean a sequence of sets $\left\{X_{n}\right\}$, which is a relabelling of the countable collection

$$
\begin{aligned}
Y_{m j}=\{x \in X: \delta(x)>1 / m\} \cap[j / m,(j+1) / m], & \\
& m=1,2,3, \ldots \text { and } j=0, \pm 1, \pm 2, \pm 3, \ldots
\end{aligned}
$$

The key features of such a decomposition of a set $X$ are:

(i) $\cup_{n=1}^{\infty} X_{n}=X$;

(ii) if $x$ and $y$ belong to the same set $X_{n}$ then $|x-y|<\min \{\delta(x), \delta(y)\}$, and

(iii) if $x \in \bar{X}_{n}$ then there are points $y \in X_{n}$ with $|x-y|<\min \{\delta(x), \delta(y)\}$.

This decomposition is used in differentiation theory in a variety of ways. To show that a set $X$ is small in some sense (e.g. countable, measure zero, first category, $\sigma$-porous), we may form an appropriate $\delta$-decomposition and establish that each set in the decomposition is small since, by (i), $X$ is the union of these sets. To establish some statement about a perfect set (e.g. to show that a function is in the first class of Baire), we may form an appropriate decomposition of the set and then choose that 
member of the decomposition that is dense in a portion of the perfect set; the feature (iii) allows a statement of some kind about that portion. It should be clear that such decompositions, in the presence of an intersection condition, will provide many results.

4. Extreme path derivates. In this section we develop a number of properties of path derivatives in the presence of some of the conditions of the previous section. These results, however, can be more clearly expressed in the language of extreme derivates, rather than exact derivatives. In subsequent sections, we will return to the study of $E$-primitives and their derivatives. Extreme path derivates are defined in the obvious way.

4.1. Definition. Let $E=\left\{E_{x}: x \in R\right\}$ be a system of paths and let $F$ be a function on $R$. Then the extreme $E$-derivates of $F$ at a point $x$ are

$$
\bar{F}_{E}^{\prime}(x)=\limsup _{\substack{y \rightarrow x \\ y \in E_{x}}} \frac{F(y)-F(x)}{y-x} \text { and } \underline{F}_{E}^{\prime}(x)=\lim _{\substack{y \rightarrow x \\ y \in E_{x}}} \frac{F(y)-F(x)}{y-x} .
$$

A number of relations are immediately obvious. A function $F$ is $E$-differentiable at a point $x$ if and only if $\underline{F}_{E}^{\prime}(x)=\bar{F}_{E}^{\prime}(x)$ are finite. If $\underline{F}^{\prime}(x)$ and $\bar{F}^{\prime}(x)$ denote the usual bilateral extreme derivates of $F$, then $\underline{F}^{\prime}(x) \leqslant \underline{F}_{E}^{\prime}(\bar{x}) \leqslant \bar{F}_{E}^{\prime}(x) \leqslant \bar{F}^{\prime}(x)$ at every point $x$. For further properties of these derivates, we are best guided, at first, by the many known relations that hold for the Dini derivatives. The first of these was obtained by G. C. Young [44] and has been generalized in a number of settings $[5,6,25]$.

4.2 Theorem. Let $E=\left\{E_{x}: x \in R\right\}$ and $E^{*}=\left\{E_{x}^{*}: x \in R\right\}$ be systems of paths, both of which satisfy the intersection condition. Then the set $\left\{x: \bar{F}_{E}^{\prime}(x)<\underline{F}_{E^{*}}^{\prime}(x)\right\}$ is, for any function $F$, at most denumerable.

Proof. The set of the theorem is the union of the sets $X^{\alpha}=\left\{x: \bar{F}_{E}^{\prime}(x)<\alpha<\right.$ $\left.F_{E^{*}}^{\prime}(x)\right\}$ for rational numbers $\alpha$, so the theorem is proved if we show that each such $X^{\alpha}$ is at most denumberable. Let $\delta$ and $\delta^{*}$ be the positive functions associated with $E$ and $E^{*}$, respectively, for the I.C. and choose further positive functions $\delta_{1}$ and $\delta_{2}$ so that

$$
\frac{F(y)-F(x)}{y-x}<\alpha \quad \text { if } x \in X^{\alpha}, y \in E_{x}, \text { and } 0<|y-x|<\delta_{1}(x),
$$

and

$$
\frac{F(y)-F(x)}{y-x}>\alpha \text { if } x \in X^{\alpha}, y \in E_{x}^{*} \text {, and } 0<|y-x|<\delta_{2}(x) .
$$

Set $\delta_{3}=\min \left\{\delta, \delta^{*}, \delta_{1}, \delta_{2}\right\}$, and let $\left\{X_{n}^{\alpha}\right\}$ be a $\delta_{3}$-decomposition of the set $X^{\alpha}$.

We claim that each set $X_{n}^{\alpha}$ contains at most a single point. If not, then there are points $x, y \in X_{n}^{\alpha}$ with $x<y$. Select, by the I.C., a point $z \in E_{x} \cap E_{y} \cap[x, y]$. If $z=x$ or $z=y$, then we have immediately that $(F(y)-F(x)) /(y-x)<\alpha$. If $x<z<y$ then we have

$$
\frac{F(z)-F(x)}{z-x}<\alpha \text { and } \frac{F(y)-F(z)}{y-z}<\alpha
$$


so, again, $(F(y)-F(x)) /(y-x)<\alpha$. Similarly, we may select a point $z \in E_{x}^{*} \cap$ $E_{y}^{*} \cap[x, y]$ and obtain that $(F(y)-F(x)) /(y-x)>\alpha$.

Thus, the assumption that $X_{n}^{\alpha}$ contains two distinct points leads to a contradiction. Hence, each $X^{\alpha}$ is at most denumerable, and the theorem follows.

The next theorem was obtained for the Dini derivatives by W. H. Young [45]. It too has received a number of generalizations $[5,6]$.

4.3 TheOREM. Let $E=\left\{E_{x}: x \in R\right\}$ be a system of paths and let $F$ be continuous. If $E$ satisfies the intersection condition, then $\underline{F}_{E}^{\prime}(x)=\underline{F}^{\prime}(x)$ and $\bar{F}^{\prime}(x)=\bar{F}_{E}^{\prime}(x)$ except, possibly, in a first category subset of $R$.

Proof. As in the preceding theorem let $X^{\alpha}$ denote the set $X^{\alpha}=\left\{x: \bar{F}_{E}^{\prime}(x)<\alpha<\right.$ $\left.\bar{F}^{\prime}(x)\right\}$ for rational numbers $\alpha$ and observe that the theorem is proved if we are able to show that $X^{\alpha}$ is first category. Let $\delta$ be the positive function associated with $E$ by the I.C. and choose a positive function $\delta_{1}$ so that $(F(y)-F(x)) /(y-x)<\alpha$ if $x \in X, y \in E_{x}, 0<|x-y|<\delta_{1}(x)$.

Set $\delta_{2}=\min \left\{\delta, \delta_{1}\right\}$ and let $\left\{X_{n}^{\alpha}\right\}$ be a $\delta_{2}$-decomposition of the set $X^{\alpha}$. The theorem follows if each set $X_{n}^{\alpha}$ is nowhere dense. If not, then some $\bar{X}_{n}^{\alpha}$ contains an interval $(c, d)$. By earlier arguments we know that $(F(y)-F(x)) /(y-x)<\alpha$ for any points $x, y \in X_{n}^{\alpha}$; then, since $F$ is continuous, we must have

$$
(F(y)-F(x)) /(y-x) \leqslant \alpha
$$

for every pair of distinct points in $(c, d)$ and, consequently, $\bar{F}^{\prime}(x) \leqslant \alpha$ everywhere in that interval. But, by assumption, $\bar{F}^{\prime}(x)>\alpha$ for those points in $X_{n}^{\alpha} \cap(c, d)$, which is a contradiction. The theorem then follows.

Our next theorem was first obtained by Mišik [22] as an expression of the equality between the four Dini derivatives and the four approximate Dini derivatives of monotonic functions. By exploiting the concept of set porosity, we are able to give conditions that are necessary, as well as sufficient, for these relations to hold.

4.4 Theorem. Let $E=\left\{E_{x}: x \in R\right\}$ be a system of paths and suppose $F$ is monotonic. Then:

(4.4.1) If $E$ is nonporous on the right at a point $x$,

$$
\underline{F}_{E}^{\prime}(x) \leqslant D_{+} F(x) \leqslant D^{+} F(x) \leqslant \bar{F}_{E}^{\prime}(x) .
$$

(4.4.2) If $E$ is nonporous on the left at a point $x$,

$$
\underline{F}_{E}^{\prime}(x) \leqslant D_{-} F(x) \leqslant D^{-} F(x) \leqslant \bar{F}_{E}^{\prime}(x) .
$$

(4.4.3) If $E$ is nonporous at a point $x$,

$$
\underline{F}_{E}^{\prime}(x)=\underline{F}^{\prime}(x) \text { and } \bar{F}_{E}^{\prime}(x)=\bar{F}^{\prime}(x) .
$$

Conversely, these relations are maintained for all monotonic functions $F$ only if the nonporosity condition is met.

Proof. The proof depends on a simple lemma due essentially to Mišik [22]. To prove it we use some ideas from Evans and Humke [13].

(4.4.4) Let $F$ be a nondecreasing function such that at a point $x, D_{+} F(x)<\alpha$. Then the set $Y=\{y:(F(y)-F(x)) /(y-x) \geqslant \alpha\}$ is porous on the right at $x$. 
To prove (4.4.4) choose a number $\beta$ so that $D_{+} F(x)<\beta<\alpha$ and set $\theta=\beta / \alpha$. Since $F$ is nondecreasing, both $\alpha$ and $\beta$ are positive. Select a sequence $\left\{h_{k}\right\}$ tending down to zero so that $\left[F\left(x+h_{k}\right)-F(x)\right] / h_{k}<\beta$ for each $k$, and observe that if $y$ belongs to any interval $\left(x+\theta h_{k}, x+h_{k}\right)$ we must have, since $F$ is nondecreasing,

$$
\frac{F(y)-F(x)}{y-x} \leqslant \frac{F\left(x+h_{k}\right)-F(x)}{h_{k}} \cdot \frac{h_{k}}{y-x}<\frac{\beta}{\theta}=\alpha .
$$

Consequently, all such intervals $\left(x+\theta h_{k}, x+h_{k}\right)$ are disjoint from the set $Y$, and it is easy to see now that $Y$ has positive porosity on the right at $x$ as required.

Returning now to the proof of the theorem, let us address just the first part of (4.4.1), as everything else follows in a similar manner. Consider that, contrary to the theorem, $\underline{F}_{E}^{\prime}(x)>D_{+} F(x)$, at a point $x$, and yet $E_{x}$ is nonporous on the right at $x$. Choose a number $\alpha$ so $\underline{F}_{E}^{\prime}(x)>\alpha>D_{+} F(x)$. Then there is a number $\delta>0$ so

$$
\frac{F(y)-F(x)}{y-x}>\alpha \text { if } y \in E_{x} \text { and } 0<y-x<\delta .
$$

Thus

$$
E_{x} \cap(x, x+\delta) \subset\left\{y: \frac{F(y)-F(x)}{y-x} \geqslant \alpha\right\} .
$$

By the lemma, since $D_{+} F(x)<\alpha$, this set is porous on the right at $x$, and hence $E_{x}$ must also be porous on the right at $x$. As this is a contradiction, our result follows.

There remains only the converse part of the theorem. Suppose $E_{x}$ is porous at a point $x$, say on the right. Then there is a number $0<\theta<1$ and a sequence $h_{k} \downarrow 0$ so that $E_{x}$ is disjoint from $\cup_{k=1}^{\infty}\left(x+\theta h_{k}, x+h_{k}\right)$. We may suppose that these intervals are disjoint. Define a function $F$ by setting $F(t)=h_{k}$ if $x+h_{k+1} \leqslant t \leqslant x$ $+\theta h_{k} ; F(t)=\theta h_{k}$ if $x+\theta h_{k}<t<x+h_{k}$, and $F(t)=0$ for all other values. Then $D_{+} F(x)=\theta<1$, and $\underline{F}_{E}^{\prime}(x) \geqslant 1$. This example completes the proof. Note that one can construct an $F$ here that is even continuous and yet violates these relations.

We turn now to a discussion of the properties a function $F$ must have if it has finite extreme path derivates on a set. Our theorems are generalizations of theorems first obtained by Denjoy (see [33, pp. 234-240]). Recall that a function $F$ is $V B G$ on a set $X$ if $X$ can be expressed as the union of a sequence of sets on each of which $F$ is of bounded variation $V B$; if the sets in the sequence can be taken as closed, $F$ is said to be $[V B G]$. Similarly, $F$ is $(A C G)$ on a set $X$ is that set can be expressed as the union of a sequence of sets on each of which $F$ is $A C$; if the sets in the sequence can be taken as closed, then $F$ is said to be $[A C G]$. Of course, if $F$ is continuous, then both of these are equivalent to the more familiar $A C G$ concept.

4.5 THEOREM. Let $E=\left\{E_{x}: x \in R\right\}$ be a system of paths satisfying the intersection condition. If everywhere in a set $X$ one of the extreme derivates $\underline{F}_{E}^{\prime}(x)$ or $\bar{F}_{E}^{\prime}(x)$ is finite, then $F$ is VBG on $X$. If, further, the set $X$ is closed, then $F$ is [VBG] on $X$.

Proof. As usual, it is enough to show that $F$ is $V B G$ on the set $X=\{x$ : $\left.\bar{F}_{E}^{\prime}(x)<+\infty\right\}$. For this, suppose $f$ is a finite function such that, everywhere in $x$, $\overline{F_{E}^{\prime}}(x)<f(x)$, and define the sequence of sets $X^{m}$ by $X^{m}=\{x \in X: f(x)<m\}$. Let 
$\delta$ be the positive function associated with $E$, by the I.C., and choose a further positive function $\delta_{1}$ so that

$$
\frac{F(y)-F(x)}{y-x}<f(x) \text { if } y \in E_{x}, x \in X, 0<|y-x|<\delta_{1}(x) .
$$

Set $\delta_{2}=\min \left\{\delta_{1}, \delta\right\}$, and let $\left\{X_{n}^{m}\right\}$ be a $\delta_{2}$-decomposition of the set $X^{m}$. Familiar arguments show for points $x, y$ in $X_{n}^{m}$ that $(F(y)-F(x)) /(y-x)<m$, and hence $F(x)-m x$ is monotonic on each set $X_{n}^{m}$. By standard arguments, $F$ must be $V B G$ on each $X_{n}^{m}$ so, since $X=\cup_{m=1}^{\infty} \cup_{n=1}^{\infty} X_{n}^{m}, F$ is $V B G$ on $X$ as required.

Now suppose $X$ is closed. Then each $\bar{X}_{n}^{m} \subset X$, and we shall show that $F$ is $V B$ on each $\bar{X}_{n}^{m}$. In fact, the inequality $(F(y)-F(x)) /(y-x) \leqslant m$ holds on $\bar{X}_{n}^{m}$. Let us prove a special case as an illustration: suppose $x$ is a limit point of $X_{n}^{m}$ and $y \in X_{n}^{m}$. Then there is a sequence of points $x_{k} \rightarrow x, x_{k} \in X_{n}^{m}$, and by the nature of the $\delta_{2}$-decomposition we must have $\left|x_{k}-x\right|<\min \left\{\delta_{2}(x), \delta_{2}\left(x_{k}\right)\right\}$ for sufficiently large $k$. For such $x_{k}$ choose a point $z_{k}$ in $E_{x} \cap E_{x_{k}} \cap\left[x, x_{k}\right]$ or $E_{x} \cap E_{x_{k}} \cap\left[x_{k}, x\right]$. If $z_{k}=x$ then certainly

$$
\left[F\left(x_{k}\right)-F(x)\right] /\left(x_{k}-x\right)<m
$$

if $z_{k}=x_{k}$ then

$$
\left[F\left(x_{k}\right)-F(x)\right] /\left(x_{k}-x\right)<f(x) ;
$$

if $z_{k}$ lies between $x$ and $x_{k}$, then

$$
\left[F\left(z_{k}\right)-F(x)\right] /\left(z_{k}-x\right)<f(x) \text { and }\left[F\left(x_{k}\right)-F\left(z_{k}\right)\right] /\left(x_{k}-z_{k}\right)<m \text {. }
$$

In any case, we then have (supposing without loss in generality that $x \leqslant z_{k} \leqslant x_{k}$ $<y)$

$$
\begin{aligned}
F(y)-F(x) & =F(y)-F\left(x_{k}\right)+F\left(x_{k}\right)-F(x) \\
& \leqslant m\left(y-x_{k}\right)+m\left(x_{k}-z_{k}\right)+f(x)\left(z_{k}-x\right),
\end{aligned}
$$

and hence, by letting $k \rightarrow+\infty$, we obtain $(F(y)-F(x)) /(y-x) \leqslant m$. Since this can be proved for any pair of points $x, y$ in $\bar{X}_{n}^{m}$, we have that $F$ must be $[V B G]$ on $X$, as required.

4.6 THEOREM. Let $E=\left\{E_{x}: x \in R\right\}$ be a system of paths satisfying any of the intersection conditions of Definition 3.3. Then if a function $F$ has both $\underline{F}_{E}^{\prime}(x)$ and $\bar{F}_{E}^{\prime}(x)$ finite everywhere in a set $X, F$ is $(A C G)$ on $X$. If, further, the set $X$ is closed, then $F$ is [ACG] on $X$.

Proof. The proof is very close in detail to that of the preceding theorem. Let us prove this under just the assumption that $E$ has a one-sided external intersection condition $(m=1)$. The other intersection conditions permit similar proofs. As before, we select a finite function $f$ so that $-f(x)<\underline{F}_{E}^{\prime}(x) \leqslant \bar{F}_{E}^{\prime}(x)<f(x)$ everywhere on a set $X$, and define $X^{m}$ as the set $X^{m}=\{x \in X: f(x)<m\}$. Let $\delta$ be that positive function associated with $E$, for the one-sided E.I.C., and choose a further positive function $\delta_{1}$ so $-f(x)<(F(y)-F(x)) /(y-x)<f(x)$ for every $x \in X$, $y \in E_{x}$, and $0<|y-x|<\delta_{1}(x)$. Set $\delta_{2}=\min \left\{\delta, \delta_{1}\right\}$, and let $\left\{X_{n}^{m}\right\}$ be a $\delta_{2}$-decomposition of the set $X^{m}$. We show that $F$ satisfies a Lipshitz condition on each set $X_{n}^{m}$, and it will follow that $F$ is $(A C G)$ on the set $X=\cup_{m=1}^{\infty} \cup_{n=1}^{\infty} X_{n}^{m}$. 
Suppose $x, y \in X_{n}^{m}$ with $x<y$; then, by the one-sided E.I.C., there is a point $z$ in one of the sets $E_{x} \cap E_{y} \cap(y, 2 y-x)$ or $E_{x} \cap E_{y} \cap(2 x-y, x)$. Suppose the former is nonempty. Then

$$
|F(z)-F(x)| \leqslant m(z-x) \leqslant m(2 y-x-x)=2 m(y-x)
$$

and

$$
|F(z)-F(y)| \leqslant m(z-y) \leqslant m(2 y-x-y)=m(y-x),
$$

which gives

$$
|F(y)-F(x)| \leqslant 3 m(y-x) .
$$

The same inequality holds if it is the other set that is nonempty. It follows then that $F$ is $(A C G)$ on $X$.

If $X$ is closed then each $\bar{X}_{n}^{m} \subset X$, and arguments similar to those used in the preceding theorem can be used to show that the Lipshitz condition is maintained on the closures of the sets $X_{n}^{m}$ so $F$ is even $[A C G]$ on $X$.

Finally, we obtain a monotonicity theorem expressible in terms of extreme path derivatives.

4.7 TheOREM. Let $E=\left\{E_{x}: x \in R\right\}$ be a system of paths that is bilateral and satisfies the intersection condition. Then, in order for a function $F$ to be nondecreasing on an interval $[a, b]$, either of the following suffice:

(4.7.1) $\underline{F}_{E}^{\prime}(x) \geqslant 0$ a.e. and $\underline{F}_{E}^{\prime}(x)>-\infty$ everywhere in $[a, b]$, or

(4.7.2) $\underline{F}_{E}^{\prime}(x)=\bar{F}_{E}^{\prime}(x)$ n.e. and $\underline{F}_{E}^{\prime}(x) \geqslant 0$ a.e. in $[a, b]$ with $F$ in the first class of Baire and satisfying the Darboux property.

Proof. Both of these are consequences of the general monotonicity theorems given in [38 and 39]. The only detail that is not immediate is that a "partitioning property" is available for this type of derivate. We will prove this here. Let us define a collection $C$ of subintervals of a fixed interval $[a, b]$ to be an $E$-full cover of $[a, b]$ if there is a positive function $\delta$ on $[a, b]$ so that every interval $[y, z]$, for which

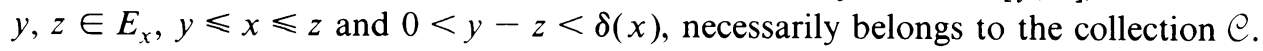
The needed result is

(4.7.3) Let $E=\left\{E_{x}: x \in R\right\}$ be a system of paths that is bilateral and satisfies the intersection condition. Then if $\mathcal{C}$ is an $E$-full cover of the interval $[a, b], \mathcal{C}$ must contain a partition of every subinterval of $[a, b]$.

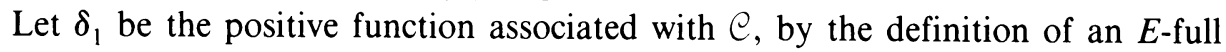
cover, and let $\delta_{2}$ be the positive function associated with $E$ by the intersection condition. Set $\delta_{3}=\min \left\{\delta_{1}, \delta_{2}\right\}$.

We obtain the existence of partitions by a standard category argument. Define $\mathcal{G}$ as the collection of all open intervals $(c, d) \subset[a, b]$ such that $\mathcal{C}$ contains a partition of every closed interval contained in $(c, d)$, and write $G=\cup\{(c, d):(c, d) \in \mathcal{G}\}$. Observe, firstly, that if $G$ contains an interval $[x, y]$ then, by a simple compactness argument, $\mathcal{C}$ must contain a partition of $[x, y]$. Secondly, if $(x, y)$ is a component interval of the (open) set $G$, the fact that $E$ is bilateral assures that $巳$ must contain a partition of $[x, y]$. 
Consequently, the result we wish to prove depends on showing that $G=(a, b)$. Suppose not; then the set $Q=[a, b] \backslash G$ must be perfect. Let $\left\{Q_{n}\right\}$ be a $\delta_{3}$-decomposition of $Q$, and then, by Baire's theorem, one of these sets is dense in a nonempty portion of $Q$. Suppose $Q_{m}$ is dense in $Q \cap(c, d)$.

We shall show that $\mathcal{C}$ contains a partition of any interval $[x, y] \subset(c, d)$, which will contradict the definition of the set $Q$. If $(x, y)$ contains no points of $Q$, then $(x, y) \subset G$, and so $\mathcal{E}$ must contain a partition of $[x, y]$ and we are done. Otherwise, define $x_{1}=\inf Q \cap(x, y)$ and $y_{1}=\sup Q \cap(x, y)$. The intervals $\left[x, x_{1}\right]$ and $\left[y_{1}, y\right]$ are either degenerate or else $\mathcal{e}$ contains a partition of each; hence we need only find a partition of $\left[x_{1}, y_{1}\right]$. Now, $x_{1}$ and $y_{1}$ are, respectively, right-hand and left-hand limit points of $Q$, and $Q_{m}$ is dense here, so we may find points $x_{2}, y_{2} \in Q_{m}$ with $x_{1}<x_{2}<y_{2}<y_{1}, 0<x_{2}-x_{1}<\delta_{3}\left(x_{1}\right)$, and $0<y_{1}-y_{2}<\delta_{3}\left(y_{1}\right)$. It remains only to show that $e$ contains partitions of each interval $\left[x_{1}, x_{2}\right],\left[x_{2}, y_{2}\right]$, and $\left[y_{2}, y_{1}\right]$.

Each of these is supplied, by I.C., in an obvious way; for example, take a point $z$ in the intersection $E_{x_{2}} \cap E_{y_{2}} \cap\left[x_{2}, y_{2}\right]$. If $z=x_{2}$ or $z=y_{2}$, then the interval $\left[x_{2}, y_{2}\right]$ is a member of $\mathcal{E}$ and we are done; if $x_{2}<z<y_{2}$ then both intervals $\left[x_{2}, z\right]$ and $\left[z, y_{2}\right]$ belong to $\mathcal{e}$, and again we have the partition.

Thus, we obtain a partition from $\mathcal{e}$ of every subinterval of $(c, d)$, and this contradiction proves (4.7.3).

5. Properties of primitives of path derivatives. We turn now to a study of ways in which the intersection conditions of $\$ 3$ require desirable behaviour of $E$-differentiable functions. In particular, we show what is behind the many properties, possessed by approximately differentiable functions, which we summarized in our introduction.

We begin by defining a notion of continuity connected with a system of paths; this would include, as special cases, many familiar generalizations of continuity, such as approximate continuity, preponderant continuity, and one-sided continuity.

5.1 Definition. Let $E=\left\{E_{x}: x \in R\right\}$ be a system of paths and let $F$ be a function. We say that $F$ is $E$-continuous at a point $x$ if

$$
\lim _{\substack{y \rightarrow x \\ y \in E_{x}}} F(y)=F(x) .
$$

If $F$ is everywhere $E$-continuous, then we say that $F$ is $E$-continuous.

Note that any continuous function is necessarily $E$-continuous for any choice of paths, and an $E$-differentiable function must be $E$-continuous.

5.2 ThEOREM. Let $E=\left\{E_{x}: x \in R\right\}$ be a system of paths that satisfies any one of the intersection conditions of Definition 3.3. Then any E-continuous function is in the first class of Baire.

Proof. By standard arguments [4, p. 104], if a function $F$ is not in the first class of Baire, there must exist a perfect set $Q$ and a positive number $\varepsilon$, so that the oscillation of $F$, on $Q$, exceeds $\varepsilon$ at every point of $Q$. Suppose such $Q$ and $\varepsilon$ exist and yet $F$ is E-continuous. 
Let $\delta_{1}$ be the positive function associated with $E$, for I.C., and choose a further positive function $\delta_{2}$ so that $|F(y)-F(x)|<\varepsilon / 6$ for $y \in E_{x}$ and $0<|y-x|<\delta_{2}(x)$. Let $\delta_{3}=\min \left\{\delta_{1}, \delta_{2}\right\}$ and let $\left\{Q_{n}\right\}$ be a $\delta_{3}$-decomposition of the set $Q$. By Baire's theorem one of these sets, say $Q_{m}$, is dense in a nonempty portion, say $Q \cap(c, d)$, of $Q$.

Let $x, y$ be any points in $Q \cap(c, d)$ with $x<y$. We may choose points $x_{m}, y_{m} \in$ $Q_{m}$ so that $\left|x-x_{m}\right|<\delta_{3}(x)$ and $\left|y-y_{m}\right|<\delta_{3}(y)$. Then by I.C. there are points $z_{m}, z_{m}^{\prime}$, and $z_{m}^{\prime \prime}$ such that each of the inequalities

$$
\begin{aligned}
& \left|F(x)-F\left(z_{m}\right)\right|<\varepsilon / 6, \quad\left|F\left(z_{m}\right)-F\left(x_{m}\right)\right|<\varepsilon / 6, \quad\left|F\left(x_{m}\right)-F\left(z_{m}^{\prime}\right)\right|<\varepsilon / 6, \\
& \left|F\left(z_{m}^{\prime}\right)-F\left(y_{m}\right)\right|<\varepsilon / 6, \quad\left|F\left(y_{m}\right)-F\left(z_{m}^{\prime \prime}\right)\right|<\varepsilon / 6, \quad\left|F\left(z_{m}^{\prime \prime}\right)-F(y)\right|<\varepsilon / 6
\end{aligned}
$$

holds. But this gives $|F(y)-F(x)|<\varepsilon$, which contradicts our choice of $Q$ and $\varepsilon$. Consequently, $F$ must be Baire 1, as required. The same proof, with slight modifications, works under any of the intersection conditions.

5.3 THEOREM. Let $F$ be in the first class of Baire and E-continuous for a choice of paths $E=\left\{E_{x}: x \in R\right\}$. If $E$ is bilateral then $F$ has the Darboux property.

Proof. This follows easily from [4, Theorem 1.1(2), p. 9]. In fact, it is also easy to see that $E$ must be bilateral for this to hold in general.

These theorems apply, of course, to $E$-differentiable functions, but, in fact, much more can be said for these. Such functions, in the presence of an intersection condition, are even $[A C G]$, and so $B_{1}^{*}$ (in the language of $[24]$ ) or $[C]$ (in the language of [1]).

5.4 THEOREM. Let $E=\left\{E_{x}: x \in R\right\}$ be a system of paths satisfying any of the intersection conditions of Definition 3.3. Then any E-differentiable function is $[A C G]$.

Proof. This is just a special case of Theorem 4.6, since we always assume that our derivatives, if they exist, are finite.

5.5 Corollary. Let $E=\left\{E_{x}: x \in R\right\}$ be a system of paths satisfying any of the intersection conditions and let $F$ be an E-differentiable function. Then there exists $a$ sequence of intervals $\left\{I_{k}\right\}$ whose union is dense in $R$ and $F$ is absolutely continuous on each $I_{k}$. In particular, $F$ is differentiable a.e. on a dense open set and is approximately differentiable a.e. on $R$.

This will apply to a great many derivatives, as has been observed before. For preponderant derivatives see [3] and for selective derivatives see [25]. The approximately differentiable functions permit a sharpening of this result which, in our setting, appears in Theorem 7.6 below.

5.6 Corollary. If $E=\left\{E_{x}: x \in R\right\}$ is a system of paths satisfying any of the intersection conditions, then any Darboux E-differentiable function possesses local extrema on compact intervals. 
Proof. Since such functions are $[A C G]$ this follows from [24].

Note that these corollaries are not valid if the function is assumed merely to be $E$-continuous. For example, even approximately continuous functions need not assume local extrema on any compact intervals (see [24]).

6. Properties of path derivatives. It has been known for a long time that ordinary derivatives possess the Darboux property and are in the first class of Baire. Somewhat less well known is that derivatives also possess the Denjoy property: if $\alpha<F^{\prime}\left(x_{0}\right)<\beta$ then $\left\{x: \alpha<F^{\prime}(x)<\beta\right\}$ has positive measure in every neighborhood of the point $x_{0}$. In his deep study of derivatives, Zahorski [46] showed that derivatives posses still stronger properties, which he labelled $\mathfrak{M}_{2}$ and $\mathfrak{N}_{3}$.

It had been known for some time that approximate derivatives also possessed some of these same properties, but it was not until 1965 that the $\mathfrak{N}_{3}$ property was obtained [41]. In recent years, other generalized derivatives have been shown to share these same properties, at least in the presence of other conditions $[\mathbf{2 3}, \mathbf{2 7}, \mathbf{3 2}$, and 41].

Here we shall show how the basic conditions, defined in $\$ 3$, can be used to establish all of these properties for path derivatives. Some of our theorems provide new and simpler proofs of known results, as well as giving a deeper insight into why various properties must hold. For example, it is known that preponderant derivatives are in $\mathscr{\Omega}_{2}$, but may fail to be in $\mathscr{N}_{3}$. Theorem 6.10 shows exactly what is lacking in the paths and provides a method of proving that some specific instance of a preponderant derivative (which may yet fail to be an approximate derivative) will belong to $\Re_{3}$.

Our first result establishes that a path derivative is, under the appropriate conditions, in the first class of Baire. This is obtained by generalizing, to our setting, a theorem of Gleyzal [14] on convergent interval functions.

6.1 Definition. Let $E=\left\{E_{x}: x \in R\right\}$ be a system of paths, and let $\varphi$ be a real-valued interval function. We say that $\varphi$ converges to a function $f$ along $E$ if for every positive number $\varepsilon$ there is a positive function $\delta$ so that $|\varphi([y, z])-f(x)|<\varepsilon$ whenever $y, z \in E_{x}, y \leqslant x \leqslant z$, and $0<z-y<\delta(x)$.

6.2 Theorem. Let $E=\left\{E_{x}: x \in R\right\}$ be a system of paths that has the external intersection property (E.I.C. $[\mathrm{m}])$. Then if $\varphi$ is an interval function converging to a function $f$ along $E, f$ must be Baire 1.

Proof. We need a two sided E.I.C. with some choice of parameter $m$. For simplicity let us take $m=1$. As in the proof of Theorem 5.2, we obtain a contradiction by supposing, contrary to the theorem, that there is a perfect set $Q$ and a positive number $\varepsilon$, so that the oscillation of $f$ on $Q$ exceeds $\varepsilon$ at every point of $Q$.

Let $\delta_{1}$ be that positive function associated with $E$, by the E.I.C., and choose a further positive function $\delta_{2}$ so that $|\varphi([y, z])-f(x)|<\varepsilon / 6$ if $y, z \in E_{x}, y \leqslant x \leqslant z$ and $0<z-y<3 \delta_{2}(x)$. Let $\delta_{3}=\min \left\{\delta_{1}, \delta_{2}\right\}$, and let $\left\{Q_{n}\right\}$ be a $\delta_{3}$-decomposition of the set $Q$. Using Baire's theorem we may pass to a portion $Q \cap(c, d)$ and a set $Q_{m}$ dense in that portion. 
Let $x, y \in Q \cap(c, d)$. Then since $Q_{m}$ is dense in $Q \cap(c, d)$, we may choose points $x_{1}, y_{1} \in Q_{m}$ so that $\left|x-x_{1}\right|<\delta_{3}(x)$ and $\left|y-y_{1}\right|<\delta_{3}(y)$. By E.I.C. there are points $a$ and $b$ so that $a<x<b, a<x_{1}<b, a, b \in E_{x}, a, b \in E_{x_{1}}, b-a<3 \delta_{2}(x)$, and $b-a<3 \delta_{2}\left(x_{1}\right)$. This means $|\varphi([a, b])-f(x)|<\varepsilon / 6$ and $\left|\varphi([a, b])-f\left(x_{1}\right)\right|<$ $\varepsilon / 6$ so $\left|f(x)-f\left(x_{1}\right)\right|<\varepsilon / 3$. Similar arguments give $\left|f\left(x_{1}\right)-f\left(y_{1}\right)\right|<\varepsilon / 3$ and $\mid f\left(y_{1}\right)$ $-f(y) \mid<\varepsilon / 3$.

Consequently, we must have $|f(x)-f(y)|<\varepsilon$ for any choice of points $x$ and $y$ from $Q \cap(c, d)$, which contradicts our choice of $Q$ and $\varepsilon$. This contradiction proves the theorem.

6.3 Corollary. Let $E=\left\{E_{x}: x \in R\right\}$ be a system of paths satisfying the external intersection property (E.I.C. $[m])$. Then any E-derivative is Baire 1.

Proof. This follows directly from the theorem, since if $F_{E}^{\prime}(x)=f(x)$ then $f$ is the limit of the convergent interval function $\varphi([y, z])=[F(z)-F(y)] /(z-y)$ along E.

As a consequence of this corollary, we immediately have that approximate derivatives are in the first class of Baire. There have been a number of proofs for this fact. The first of these was given by Tolstoff [37] and is quite complicated; subsequent proofs by Goffman and Neugebauer [15] and by O'Malley [26] are simpler but less accessible than that presented here. Note that this extends immediately to preponderant type derivatives along paths of type $(c, c)$ density for any $c>\frac{1}{2}$ and, with a few minor modifications, can be used to prove the strict case for $c=\frac{1}{2}$. Selective derivatives are, in general, not Baire 1 (see [25]), though they must be Baire 2 (see [19]); consequently, selective derivatives will not always be realizable as path derivatives relative to systems satisfying an external intersection condition. Peano derivatives have been proved to be Baire 1 (see [23]), but, as it is unknown whether they can be realized as path derivatives relative to systems satisfying the E.I.C., we cannot say whether this fact can follow from Corollary 6.3.

In the sequel we shall of ten assume that a path derivative is given to be Baire 1 . Since this can happen without $E$ satisfying an E.I.C., this allows us more flexibility than is possible by carrying throughout an assumption that $E$ satisfies this condition. Often one can tell by inspection that a derivative is Baire 1; for example, if the primitive is continuous then the nature of the paths may be enough to obtain this. Certainly, congruent type paths ensure that this is the case. Denjoy [10] showed that if a continuous function has a unique bilateral derived number at each point, then that bilateral path derivative must be Baire 1 .

6.4 Theorem. Let $E=\left\{E_{x}: x \in R\right\}$ be a system of paths that is bilateral and satisfies the intersection condition. If $f$ is an exact E-derivative and is Baire 1, then $f$ has the Darboux property.

Proof. Since $f$ is Baire 1, the proof may be reduced to showing the impossibility of the following situation: there is an interval $[c, d]$ and a positive number $t$ so that $f(x)>f(c)+t$ for all $x \in(c, d]$ (see [4, Theorem 1.1, p. 9]). Let $\varepsilon<t$ be an 
arbitrary positive number and choose a positive function $\delta$ so that

$$
(F(y)-F(x)) /(y-x)>f(x)-\varepsilon / 2>f(c)+t / 2
$$

for every $y \in E_{x}, x \in(c, d], 0<|y-x|<\delta(x)$ and $\delta(c)<\varepsilon$ with

$$
(F(y)-F(c)) /(y-c)>f(c)-\varepsilon \text { for } y \in E_{c} \text { and } 0<y-c<\delta(c) .
$$

Let $e$ be the collection of all intervals $[x, y]$ or $[c, z]$ with $c<x<y \leqslant d$ and $c<z \leqslant d$ such that

$$
\frac{F(y)-F(x)}{y-x}>f(c)+\frac{t}{2} \quad \text { and } \quad \frac{F(z)-F(c)}{z-c}>f(c)-\varepsilon .
$$

This collection $\mathcal{E}$ is an $E$-full cover of the interval $[c, d]$ (recall the definition preceding (4.7.3)) and so, by (4.7.3), contains a partition of any subinterval of $[c, d]$. Consider any interval $\left[c, c^{\prime}\right]$ with $c+\varepsilon<c^{\prime} \leqslant d$ : then there is in $e$ a partition $\left\{I_{i}\right\}_{i=1}^{n}$ of $\left[c, c^{\prime}\right]$ such that one of the intervals (say $I_{1}$ ) has $I_{1}=\left[c, c^{\prime \prime}\right]$ with $c^{\prime \prime}<c+\varepsilon$. This gives

$$
\begin{aligned}
F\left(c^{\prime}\right)-F(c) & =\sum_{1}^{n} F\left(I_{i}\right)=F\left(I_{1}\right)+\sum_{2}^{n} F\left(I_{i}\right) \\
& \geqslant(f(c)-\varepsilon)\left(c^{\prime \prime}-c\right)+(f(c)+t / 2)\left(c^{\prime}-c^{\prime \prime}\right) .
\end{aligned}
$$

Here $c<c^{\prime \prime}<c+\varepsilon$ and $\varepsilon$ is arbitrary; it follows then that, in fact,

$$
F\left(c^{\prime}\right)-F(c) \geqslant(f(c)+t / 2)\left(c^{\prime}-c\right)
$$

for every $c<c^{\prime} \leqslant d$. This requires, however, that $F_{E}^{\prime}(c) \geqslant f(c)+t / 2$, which contradicts the fact that $f(c)=F_{E}^{\prime}(c)$. This contradiction establishes the theorem.

This theorem, along with 6.3 , shows that every approximate derivative, and every preponderant derivative, is Darboux Baire 1. Note that the assumption that $E$ is bilateral cannot be dropped, for if there is a single point $x_{0}$ at which $E_{x_{0}}$ is not bilateral, then the function $F(x)=\left|x-x_{0}\right|$ is necessarily $E$-differentiable for any such $E$, and, clearly, $F_{E}^{\prime}$ cannot be Darboux continuous. However, in the presence of other assumptions, the intersection condition is not necessary. By a theorem of Denjoy [10], any function $f$, for which $f(x)$ is a bilateral derived number of a continuous function $F$ at $x$, must be Darboux (even though it need not be Baire 1). Thus, for continuous primitives $F$, a derivative $F_{E}^{\prime}$ will be Darboux, provided only that $E$ is bilateral. This means, too, that congruent and sequential-congruent type derivatives of continuous functions are Darboux Baire 1 on the assumption that the paths are bilateral. For the sequential-congruent derivatives, this has been observed by Petruska and Laczkovich [32].

We turn now to the Denjoy property. Evidently, the fact that a derivative $f$ be Darboux Baire 1 is not enough to conclude that it possesses the Denjoy property. (By [46] such functions need not have this property, and by 3.1 any such function can be realized as a derivative along appropriate paths.) Nevertheless, it is somewhat surprising that every known path derivative, which is Darboux Baire 1, also has the Denjoy property. When taken together with Theorems 4.7, 6.3, and 6.4, Theorem 6.7, below, offers some insight into this phenomenon. 
One of the keys to the Denjoy property is a monotonicity property. Rather than impose conditions on the system $E$, so that such a monotonicity theorem is available, we retain more flexibility by taking this as a definition. In this way, our Theorem 6.7 has a larger range of applicability.

6.5 Definition. Let $E=\left\{E_{x}: x \in R\right\}$ be a system of paths and $F$ a function on $R$. We say that $F$ has the monotonicity property relative to $E$ if on any interval $[a, b]$ the conditions $F_{E}^{\prime}(x)$ exists a.e. in $[a, b]$ and $F_{E}^{\prime}(x) \geqslant \alpha$ a.e. in $[a, b]\left[\operatorname{resp} . F_{E}^{\prime}(x) \leqslant \alpha\right]$ imply that the function $F(x)-\alpha x$ [resp. $\alpha x-F(x)]$ is nondecreasing on $[a, b]$.

Our next theorem summarizes some situations in which a monotonicity property is available.

6.6 THEOREM. Let $E=\left\{E_{x}: x \in R\right\}$ be a system of paths and let $F$ be a function. If any of the following hold, then $F$ has the monotonicity property relative to $E$ :

(6.6.1) $E$ is bilateral and satisfies the intersection condition, and $F$ is $E$-differentiable.

(6.6.2) $E$ satisfies the intersection condition and $F$ is Darboux and $[A C G]$.

(6.6.3) $E$ is bilateral and satisfies the intersection condition, and $F$ is Darboux Baire 1 with $\underline{F}_{E}^{\prime}(x)=\bar{F}_{E}^{\prime}(x)$ n.e.

(6.6.4) $E$ is bilateral (or merely unilateral but from an invariable side), and $F$ is continuous with finite E-derivates n.e.

Proof. For (6.6.1) the monotonicity property follows directly from Theorem (4.7.1), and for (6.6.3) it follows from (4.7.2).

For (6.6.4) we need only apply a classical monotonicity theorem for the Dini derivatives of a continuous function (see, for example, [4, p. 189]).

Finally, (6.6.2) follows from a monotonicity result of Ellis [12], since in the presence of an intersection condition, a derivative $F_{E}^{\prime}(x)$ will only differ from the derivative $F_{\mathrm{ap}}^{\prime}(x)$ on the set where they both exist at a denumberable number of points (this follows from Theorem 4.2).

We now state our theorem giving conditions that ensure that a derivative will possess the Denjoy property.

6.7 Theorem. Let $E=\left\{E_{x}: x \in R\right\}$ be a system of paths and let $F$ be an $E$-differentiable function that has the monotonicity property relative to $E$. If $F_{E}^{\prime}$ is Darboux Baire 1, then $F_{E}^{\prime}$ has the Denjoy property.

Proof. The standard proof (see [4, Theorem 2.1, pp. 87-88]) is easily amended to apply in this situation.

6.8 Corollary. Let $E=\left\{E_{x}: x \in R\right\}$ be a system of paths that is bilateral and satisfies both the intersection condition and the external intersection condition (E.I.C. $[m])$. Then any E-derivative has the Denjoy property.

6.9 REMARK. Zahorski's property $\mathfrak{T}_{2}$ is closely related to the Denjoy property. A function $f$ is in the class $\mathfrak{M}_{2}$ if all sets of the form $A^{\beta}=\{x: f(x)<\beta\}$ and $A_{\alpha}=\{x$ : $f(x)>\alpha\}$ are of type $F_{\sigma}$ and have positive measure in any unilateral neighbourhood of each of their members. For Baire 1 functions with the Darboux property, the Denjoy property and $\mathfrak{K}_{2}$ coincide. This is easy to see once one observes that a 
function $f$ is Darboux Baire 1 if and only if each set of the form $A^{\beta}$ or $A_{\alpha}$ is an $F_{\sigma}$ that is bilaterally dense-in-itself [46]. See the material in [4, Chapter VI] for an extensive discussion of the Zahorski classes.

We turn now to the next Zahorski class ơ ${ }_{3}$. Zahorski [46] obtained this property for ordinary derivatives, and Weil [41] extended it to approximate derivatives. No other derivative of a weaker density type (e.g. the preponderant) has this property. In order to see why this is the case, and indeed, to characterize those path derivatives that are $\mathscr{M}_{3}$, we need to view $\mathfrak{M}_{3}$ within the context of set porosity. A moment's reflection will reveal that any function $f$ that already possesses the $\mathfrak{T}_{2}$ property will have the stronger $)_{3}$ property if and only if each of the associated sets $A^{\beta}$ and $A_{\alpha}$ is nonporous at each of its members. Thus, our next theorem completely describes the setting in which a path derivative can have the $\mathcal{O N}_{3}$ property.

6.10 TheOREM. Let $E=\left\{E_{x}: x \in R\right\}$ be a system of paths and $F$ an E-differentiable function that has the monotonicity property relative to $E$ and for which $F_{E}^{\prime}$ has the Darboux property. Suppose that, at a point $x, \alpha<F_{E}^{\prime}(x)<\beta$. Then if $E$ is nonporous on the right (left) at $x$, the set $A_{\alpha}^{\beta}=\left\{z: \alpha<F_{E}^{\prime}(z)<\beta\right\}$ is also nonporous on the right (left) at $x$. Conversely, if $E_{x}$ is porous on the right (left) at $x$, then a function $F$ can be found that is continuous on $R$, continuously differentiable on $R \backslash\{x\}$, and for which $F_{E}^{\prime}$ exists and belongs to $\mathfrak{M}_{2}$, and yet the set $A_{\alpha}^{\beta}=\left\{z: \alpha<F_{E}^{\prime}(z)<\beta\right\}$ contains $x$ and is porous on the right (left) at $x$.

Proof. If $A_{\alpha}^{\beta}$ is porous on the right at $x$, we may choose a sequence $h_{n} \downarrow 0$ and a number $0<\theta<1$ so that $A_{\alpha}^{\beta}$ intersects no interval $\left[x+\theta h_{n}, x+h_{n}\right]$. We obtain a contradiction from the fact that $E_{x}$ is supposed to be nonporous on the right at $x$, and this contradiction supplies the first part of the theorem. We assume $\beta \geqslant 0$.

On each interval $\left[x+\theta h_{n}, x+h_{n}\right]$ we know that either $F_{E}^{\prime} \leqslant \alpha$ or $F_{E}^{\prime} \geqslant \beta$ since, by hypothesis, $F_{E}^{\prime}$ is Darboux. Thus, passing to a subsequence if necessary, we obtain a sequence $\left\{h_{n}\right\}$ so that $F_{E}^{\prime}(z) \leqslant \alpha$ for every $z \in\left[x+\theta h_{n}, x+h_{n}\right]$, or else $F_{E}^{\prime}(z) \geqslant \beta$ for such $z$. Let us suppose the latter. Since $F_{E}^{\prime}(x)<\beta$, there is an integer $k$ so that $F_{E}^{\prime}(x)<k \beta /(k+2)$.

As $E_{x}$ is given to be nonporous on the right at $x$, the set $E_{x}$ must intersect all but finitely many of the intervals $\left[x+\theta h_{n}, x+h_{n}\right]$; moreover, if we divide each of these intervals in $k+2$ equal subintervals, $E_{x}$ must even meet all but a finite number of these. In particular, we can find a sequence of intervals $J_{n}=\left[c_{n}, d_{n}\right]$, with $c_{n}, d_{n} \in$ $E_{x} \cap\left[x+\theta h_{n}, x+h_{n}\right]$, so that

$$
\left|J_{n}\right|>\frac{k}{k+2}\left|\left[x+\theta h_{n}, x+h_{n}\right]\right|=k(1-\theta) h_{n} /(k+2) \text {. }
$$

On each interval $J_{n}$ we know that $F_{E}^{\prime}(z) \geqslant \beta$ so that, by the monotonicity property, $F\left(J_{n}\right) \geqslant \beta\left|J_{n}\right|$.

Write $I_{n}=\left[x, c_{n}\right]$ and $K_{n}=\left[x, d_{n}\right]$ and note that $F\left(K_{n}\right)=F\left(I_{n}\right)+F\left(J_{n}\right)$; this gives

$$
\frac{F\left(K_{n}\right)}{\left|K_{n}\right|}=\frac{F\left(I_{n}\right)}{\left|I_{n}\right|} \cdot \frac{\left|I_{n}\right|}{\left|K_{n}\right|}+\frac{F\left(J_{n}\right)}{\left|J_{n}\right|} \cdot \frac{\left|J_{n}\right|}{\left|K_{n}\right|} .
$$


Now we know that $F\left(K_{n}\right) /\left|K_{n}\right| \rightarrow F_{E}^{\prime}(x)$ and $F\left(I_{n}\right) /\left|I_{n}\right| \rightarrow F_{E}^{\prime}(x)$, and we have $\left|I_{n}\right| /\left|K_{n}\right| \geqslant \theta h_{n} / h_{n}=\theta$ and $\left|J_{n}\right| /\left|K_{n}\right| \geqslant k(1-\theta) /(k+2)$. Combining these facts gives

$$
F_{E}^{\prime}(x) \geqslant \theta F_{E}^{\prime}(x)+\beta(1-\theta) k /(k+2),
$$

and hence $F_{E}^{\prime}(x) \geqslant \beta k /(k+2)$, contrary to our choice of $k$. This contradiction establishes the first part of the theorem.

For the final part of the theorem, suppose $E_{x}$ is porous on the right at $x$, and let us simplify the computations by taking $x=0$. Then there is a sequence $h_{n} \downarrow 0$ and a number $0<\theta<1$ so that $E_{0} \cap\left[\theta h_{n}, h_{n}\right]=\varnothing$, for all $n$. By passing to a subsequence if necessary, we may suppose $h_{n+1}<\theta h_{n}$.

Let $\theta<\theta_{1}<\theta_{2}<1$ be chosen and construct a function $g$, continuous except at 0 , so that $g(z)=2$ if $z \in\left[\theta_{1} h_{n}, \theta_{2} h_{n}\right], g(z)=0$ if $z$ belongs to no interval $\left[\theta h_{n}, h_{n}\right]$, and $\int g$ vanishes on each of these intervals. Define $F(z)=\int_{0}^{z} g(t) d t$. Certainly, $F_{E}^{\prime}(z)=g(z)$ everywhere, and yet the set $\{z:-1<g(z)<1\}$ is porous on the right at 0 . This function $F$ is the promised example, so the theorem is proved.

6.11 Corollary. Let $E=\left\{E_{x}: x \in R\right\}$ be a system of paths and suppose $F$ is an $E$-differentiable function that has the monotonicity property relative to $E$. Then if $E$ is nonporous and $F_{E}^{\prime}$ is Darboux Baire 1, $F_{E}^{\prime}$ must have Zahorski's $\mathfrak{M}_{3}$ property. Indeed, every set of the form $A_{\alpha}^{\beta}=\left\{\alpha<F_{E}^{\prime}(x)<\beta\right\}$ is in the class $M_{3}$.

6.12 Corollary. Let $E=\left\{E_{x}: x \in R\right\}$ be a system of paths that is nonporous and satisfies I.C. and E.I.C. Then every E-derivative has the $\mathfrak{M}_{3}$ property, and every set of the form $A_{\alpha}^{\beta}=\left\{x: \alpha<F_{E}^{\prime}(x)<\beta\right\}$ for an E-differentiable function $F$ is in the class $M_{3}$.

7. Differentiability criteria. The literature abounds with theorems which assert that a function differentiable in some generalized sense and meeting certain conditions must be, in fact, differentiable in the ordinary sense at some given point or on some substantial set. For example, a bounded approximate derivative is just an ordinary derivative; if a function has a Dini derivative that is continuous at a point, then that function is differentiable there. Our Theorem 5.4 and its corollary provide ordinary differentiability a.e. on a dense open set under mild hypotheses. In this section we obtain further results of this type. In general, of course, $E$-differentiability need imply nothing about ordinary differentiability (cf. Theorem 3.1).

7.1 TheOREM. Let $E=\left\{E_{x}: x \in R\right\}$ be a system of paths and $F$ an E-differentiable function that has the monotonicity property relative to $E$. Then if $F_{E}^{\prime}$ is continuous at a point $x, F$ is, in fact, differentiable there and $F_{E}^{\prime}(x)=F^{\prime}(x)$.

Proof. Given $\varepsilon>0$ choose an interval $(a, b)$ containing the point $x$ so that $F_{E}^{\prime}(x)-\varepsilon<F_{E}^{\prime}(z)<F_{E}^{\prime}(x)+\varepsilon$ for all $z$ in the interval $(a, b)$. Using $\alpha=F_{E}^{\prime}(x)-\varepsilon$ and $\beta=F_{E}^{\prime}(x)+\varepsilon$, we have $F_{E}^{\prime}(z) \geqslant \alpha$ and $F_{E}^{\prime}(z) \leqslant \beta$ everywhere in that interval, which, by the monotonicity property, requires $F(z)-\alpha z$ and $F(z)-\beta z$ to be, 
respectively, nondecreasing and nonincreasing. From this we obtain

$$
F_{E}^{\prime}(x)-\varepsilon=\alpha \leqslant \frac{F(y)-F(z)}{y-z} \leqslant \beta=F_{E}^{\prime}(x)+\varepsilon
$$

everywhere in that interval. The theorem now follows easily.

7.2 Corollary. If $E=\left\{E_{x}: x \in R\right\}$ is a system of paths that is bilateral and satisfies the intersection condition, then for any E-differentiable function $F$, if $x$ is a point of continuity of $F_{E}^{\prime}$ it is a point of differentiability. of $F$.

Proof. By (6.6.1) such an $F$ has the monotonicity property relative to $E$.

7.3. Corollary. If $E$ and $F$ satisfy the hypotheses of the theorem and $F_{E}^{\prime}$ is Baire 1 , then $F$ must be differentiable on a dense set of type $G_{\delta}$.

This theorem, together with its corollaries, shows that, under a number of simple hypotheses, any path derivative must actually be an ordinary derivative on a set that is large in a certain topological sense. Our next two theorems further examine the set of differentiability points for such functions.

7.4 TheOREM. Let $E=\left\{E_{x}: x \in R\right\}$ be a system of paths satisfying the intersection condition and let $F$ be a continuous E-differentiable function. Then $F$ must be differentiable in the ordinary sense on a residual set.

Proof. This follows directly from Theorem 4.3.

7.5 TheOREM. Let $E=\left\{E_{x}: x \in R\right\}$ be a system of paths that is nonporous on the right (left) at a point $x$. If $F$ is a function monotonic in a neighbourhood of $x$ and $E$-differentiable at $x$, then $F$ is even differentiable from the right (left) at $x$ and $F_{+}^{\prime}(x)=F_{E}^{\prime}(x)\left(F_{-}^{\prime}(x)=F_{E}^{\prime}(x)\right)$.

Proof. This follows directly from 4.4.

For approximate derivatives this result dates back to Khintchine [18]. Świątkowski [36] has proved an essentially equivalent theorem, stated in the language of filter derivatives, and Petruska and Laczkovich [32] give a version appropriate to path derivatives of sequential-congruent type.

It should be apparent that nonporosity of the paths, in the presence of other properties, must say a great deal about the differentiability behaviour of $E$-differentiable functions. For example, if $F$ has the monotonicity property relative to $E$, and $F_{E}^{\prime}$ is bounded on some interval $[a, b]$, it follows that $F$ will be differentiable at any point of $[a, b]$ at which the path is nonporous. Similarly, if we know that $F_{E}^{\prime}$ is Baire 1 (rather than bounded) we can produce a sequence of intervals, whose union is dense in $R$, on each of which the same will be true. There are numerous theorems possible; the one we now state can be considered a direct generalization of the corresponding assertion for approximate derivatives.

7.6 TheOREM. Let $E=\left\{E_{x}: x \in R\right\}$ be a system of paths that is nonporous and satisfies both I.C. and E.I.C. If $F$ is E-differentiable then there exists a sequence of open intervals $\left\{I_{k}\right\}$, whose union is dense in $R$, on each of which $F$ is differentiable and $F^{\prime}=F_{E}^{\prime}$. 
Proof. This follows immediately from the preceding discussion.

Note that Theorem 7.6 presents a considerable improvement over the result we obtained above in Theorem 5.5, in that, with a nonporosity assumption, $F$ is actually differentiable on a dense open subset of $R$ rather than merely a.e. differentiable on a dense open set.

7.7 REMARK. We have remarked above on conditions that ensure that a bounded $E$-derivative must be an ordinary derivative. A similar argument will reveal that, under these assumptions, if an $E$-derivative is merely dominated by an ordinary derivative, then it must itself be an ordinary derivative. To see this, let $F_{E}^{\prime}=f$ be an $E$-derivative and $g=G^{\prime}$ an ordinary derivative with $f \leqslant g$. Then $H=G-F$ is $E$-differentiable and $H_{E}^{\prime} \geqslant 0$. Thus, under any monotonicity assumption, $H$ is nondecreasing and so, assuming nonporosity, differentiable in the ordinary sense. Clearly it follows that $F$ too is differentiable.

We now conclude this section with a few further results that continue this same theme of when differentiability in some sense implies differentiability in another sense.

7.8 TheOREM. Let $E=\left\{E_{x}: x \in R\right\}$ and $E^{*}=\left\{E_{x}^{*}: x \in R\right\}$ be two systems of paths, both of which satisfy the intersection condition. If a function is both E-differentiable and $E^{*}$-differentiable on a set $X$, then $\left\{x \in X: F_{E}^{\prime}(x) \neq F_{E^{*}}^{\prime}(x)\right\}$ is at most denumerable.

Proof. This follows directly from Theorem 4.2.

7.9 COROLlary. Let $E=\left\{E_{x}: x \in R\right\}$ be a system of paths satisfying the intersection condition. If $F$ is E-differentiable a.e. on a set $X$, then $F$ is approximately differentiable a.e. on that set, and $F_{E}^{\prime}(x)=F_{\mathrm{ap}}^{\prime}(x)$ a.e. in $X$.

Proof. By Theorem 4.5 we know that $F$ is $V B G$ on any set on which it is $E$-differentiable, so it must be approximately differentiable a.e. on such a set. But the approximate derivative (which is a path derivative satisfying I.C.) cannot differ from $F_{E}^{\prime}(x)$ at more than a countable number of points on the set at which they both exist because of the theorem.

7.10 THEOREM. Let $F$ be Baire 1. Then $F$ is differentiable if and only if there is a choice of paths $E=\left\{E_{x}: x \in R\right\}$ for which $F$ is $E$-differentiable, with each $E_{x}$ dense in some neighborhood of $x$.

Proof. We omit the trivial proof.

7.11 COROLlary. A qualitatively differentiable function [20] is differentiable.

Proof. This follows directly from the theorem along with Theorem 5.2.

8. Miscellaneous results. In this section we collect some further observations about the behaviour of path derivatives under our assumptions. The first of these concerns the oscillatory nature of a path derivative that is not also an ordinary derivative and is closely related to the results, obtained in [30], for the approximate derivatives and for Peano derivatives. 
Recall that a function $f$ that is the approximate derivative of a function $F$ on an interval $[a, b]$ is, in fact, the ordinary derivative of $F$ on a dense open set $\Delta_{F} \subset[a, b]$. If there are points in $[a, b]$ at which $F$ is not differentiable, then $f$ must be unbounded both above and below on that interval; in fact, $f$ must be unbounded both above and below on $\Delta_{F}$. The result, in [30], shows more about the nature of this oscillation: given any number $M$ there must be a component interval of $\Delta_{F}$ on which $f$ assumes every value between $-M$ and $M$. This has a number of useful consequences. Here we show that several of our assumptions are sufficient to obtain a generalized version of this phenomenon.

8.1 THEOREM. Let $E=\left\{E_{x}: x \in R\right\}$ be a nonporous system of paths satisfying the intersection condition. Suppose that $F$ is an E-differentiable function with $F_{E}^{\prime}$ Baire 1. Then if $F_{E}^{\prime}$ attains the values $M$ and $-M$ on an interval $I_{0}$, there is a subinterval $I$ of $I_{0}$ on which $F$ is differentiable and $F^{\prime}$ attains both values $M$ and $-M$.

Proof. The proof follows the lines in [30, Theorems 4.1 and 4.2] and, in particular, uses the key lemma [30, Lemma 3.1, p. 468]. Note that our assumptions on $E$, that it is nonporous and satisfies the I.C., immediately give us a number of facts. From Theorem 6.4 we know that $F_{E}^{\prime}$ has the Darboux property, from (6.6.1) we have a monotonicity property for $F$ relative to $E$, and from Theorem 7.6 (but without E.I.C. since we are assuming here that $F_{E}^{\prime}$ is Baire 1) the set of points $\Delta_{F}=\operatorname{int}\left\{x \in I_{0}: F^{\prime}(x)\right.$ exists $\}$ is a dense open subset of $I_{0}$.

We wish to show that there is a component of $\Delta_{F}$ on which $F_{E}^{\prime}$ assumes the values $M$ and $-M$. Suppose, contrary to the theorem, that this is not the case; then, since $F_{E}^{\prime}$ has the Darboux property, on any component interval $(a, b)$ of the set $\Delta_{F}$ we must have either $F^{\prime}(z)>-M$ or $F^{\prime}(z)<M$ for every $z$ in $(a, b)$.

Define the set $P=I_{0} \backslash \Delta_{F}$. It is easy to see that $P$ is nonempty and closed. In fact, $P$ is perfect. To see this, it is enough to observe that in each interval $[a, b]$, contiguous to $P$ in $I_{0}$, either $F(z)+M z$ or $M z-F(z)$ is nondecreasing, so, by Theorem 7.5, the one-sided derivatives $F_{+}^{\prime}(a)$ and $F_{-}^{\prime}(b)$ must exist and agree with $F_{E}^{\prime}(a)$ and $F_{E}^{\prime}(b)$, respectively.

Since $F_{E}^{\prime}$ is given to be Baire 1 , there must be points in $P$ at which $F_{E}^{\prime}$ is continuous relative to $P$. We claim that at such a point $x_{0},\left|F_{E}^{\prime}\left(x_{0}\right)\right| \leqslant M$. For suppose that $F_{E}^{\prime}\left(x_{0}\right)>M$ (a similar argument holds if $\left.F_{E}^{\prime}\left(x_{0}\right)<-M\right)$. Then there is an open interval $I$, containing $x_{0}$, for which $F_{E}^{\prime}(x)>M$ for every $x \in I \cap P$. On the intervals $(a, b)$, that are complementary to $P$ in $I$, we know that $F_{E}^{\prime}(x)<M$ or $F_{E}^{\prime}(x)>-M$. As $F_{E}^{\prime}$ is Darboux, this means that on every such interval it must be the case that $F_{E}^{\prime}(x)>-M$, so, in fact, $F_{E}^{\prime}(x)>-M$ everywhere in $I$. By the monotonicity property, $F(z)+M z$ is nondecreasing on $I$, so, by Theorem 7.5 , it follows that $F$ is even differentiable on $I$. This contradicts the fact that $x_{0} \in I \cap P$ and proves our claim.

Let $\delta_{1}$ be the positive function associated with $E$ by the intersection condition. Let $\delta_{2}$ be a positive function chosen so that

$$
\left|(F(y)-F(x)) /(y-x)-F_{E}^{\prime}(x)\right|<1
$$


for every $y \in E_{x}$ with $0<|y-x|<\delta_{2}(x)$. Let $\delta_{3}$ be a positive function chosen so that, if $0<t<\delta_{3}(x)$, then any interval $I \subset(x-t, x+t)$ with $|I| \geqslant t / 3$ must have $I \cap E_{x} \neq \varnothing$ (this is just the porosity condition on the sets $\left\{E_{x}\right\}$ ). Write $\delta_{4}=$ $\min \left\{\delta_{1}, \delta_{2}, \delta_{3}\right\}$ and let $\left\{P_{n}\right\}$ be a $\delta_{4}$-decomposition of $P$.

Using the facts that (i) $F_{E}^{\prime}$ is Baire 1 , and (ii) the sets $P_{n}$ cover $P$, we may find a nonempty portion $P \cap(c, d)$ so that (i) $\left|F_{E}^{\prime}(x)\right|<M+1$ for every $x \in P \cap(c, d)$ (this is possible because of the discussion in the preceding paragraph), and (ii) some set $P_{m}$ (say) is dense in $P \cap(c, d)$.

The proof is completed by showing that $F$ must be differentiable at every point of the interval $(c, d)$, which contradicts the fact that it contains points of $P$. This contradiction then establishes the theorem.

The differentiability is obtained by verifying that the conditions of Lemma 3.1 of [30] hold at any point in $P \cap(c, d)$. Suppose $x \in P \cap(c, d), \lambda=F_{E}^{\prime}(x)$, and $\varepsilon>0$ is given. Then there must exist a number $\delta>0$ so that, whenever $0<|y-x|<\delta$, there are numbers $y_{1}<y<y_{2}$ with

$$
\left|F\left(y_{i}\right)-F(x)-\lambda\left(y_{i}-x\right)\right|<\varepsilon\left|y_{i}-x\right| \quad(i=1,2),
$$

and

$$
\left|y_{i}-y\right|<\varepsilon|y-x| \quad(i=1,2) .
$$

$y_{1}$ and $y_{2}$ are merely chosen from $E_{x}$ : if such choices cannot be made, it is easy to check that $E_{x}$ would then be porous at $x$, contradicting the hypotheses of the theorem.

It remains to show that the choice of $y_{1}$ and $y_{2}$ can be made so that one of the conditions 3(a), 3(b), or 3(c) of [30, Lemma 3.1] is satisfied. Certainly, if $y_{1}$ and $y_{2}$ belong to the same component interval of $\Delta_{F}$, then our assumptions that $F^{\prime}<M$ or $F^{\prime}>-M$ on such intervals give immediately that $3(\mathrm{a})$ or $3(\mathrm{~b})$ must hold. If, on the other hand, $\left[y_{1}, y_{2}\right] \cap P \neq \varnothing$, then we can show that one of the two inequalities of 3(c) must hold by establishing several bounds on the difference quotient $|(F(y)-F(x)) /(y-x)|$ for various choices of $x$ and $y$ in $(c, d)$.

Firstly, if $x$ and $y$ belong to $P_{m}$ then, since $\left|F_{E}^{\prime}(x)\right|<M+1$ on $P_{m}$, standard arguments using the I.C. (cf. 4.5 or 4.6 above) provide the inequality $|(F(y)-F(x)) /(y-x)| \leqslant M+2$. Again, as in 4.6 for example, this inequality extends to the closure of $P_{m}$, so we have this inequality for any pair of points $x$ and $y$ in $P \cap(c, d)$.

Now suppose $(a, b)$ is a component interval of $\Delta_{F}$ in $(c, d)$ : we claim that for any $x \in[a, b]$,

$$
|F(x)-F(a)| \leqslant 5(M+2)(x-a)
$$

and

$$
|F(b)-F(x)| \leqslant 5(M+2)(b-x) .
$$


It will suffice to prove only the first of these inequalities in the case where $F^{\prime}<M$ on $(a, b)$. Consider first the case $(a+b) / 2 \leqslant x \leqslant b$ : then we have $0 \leqslant b-x \leqslant x$ $-a, \quad F(x)-F(a) \leqslant M(x-a), \quad F(b)-F(a) \geqslant-(M+2)(b-a)$, and $F(b)-$ $F(x) \leqslant M(b-x)$. Combining these gives

$$
\begin{aligned}
M(x-a) & \geqslant F(x)-F(a) \geqslant[F(b)-F(a)]+[F(x)-F(b)] \\
& \geqslant-(M+2)(b-a)-M(b-x) \\
& \geqslant-(M+2)[(b-x)+(x-a)]-M(b-x) \\
& \geqslant-(M+2)(2)(x-a)-M(x-a) \geqslant-3(M+2)(x-a)
\end{aligned}
$$

so $|F(x)-F(a)| \leqslant 3(M+2)(x-a)$.

The remaining case, $a<x<(a+b) / 2$, requires different handling. Set $t=x-a$ and choose a point $z$ so $z \in P_{m} \cap(a-t, a)$ (which is possible since $P_{m}$ is dense in $P \cap(c, d))$. The interval $(x, x+t)$ exceeds in length $\frac{1}{3}$ of the interval $(z, x+t)$, so, by the way in which the decomposition was chosen, there must be a point $y$ in the intersection $E_{z} \cap(x, x+t)$. This gives

$$
|(F(y)-F(z)) /(y-z)|<\left|F_{E}^{\prime}(z)\right|+1 \leqslant M+2 .
$$

Consequently, we have the following inequalities, again assuming $F^{\prime}<M$ on $(a, b)$ :

$$
\begin{gathered}
x-a \geqslant y-x, \quad x-a \geqslant a-z, \quad F(x)-F(a) \leqslant M(x-a), \\
F(z)-F(a) \geqslant-(M+2)(a-z), \\
F(y)-F(x) \leqslant M(y-x) \text { and } F(y)-F(z) \geqslant-(M+2)(y-z) .
\end{gathered}
$$

Combining these gives

$$
\begin{aligned}
M(x-a) & \geqslant F(x)-F(a) \geqslant[F(z)-F(a)]+[F(y)-F(z)]+[F(x)-F(y)] \\
& \geqslant-(M+2)(a-z)-(M+2)(y-z)-M(y-x) \\
& =-(M+2)[(a-z)+(y-x)+(x-a)+(a-z)]-M(y-x) \\
& =-5(M+2)(x-a),
\end{aligned}
$$

so $|F(x)-F(a)| \leqslant 5(M+2)(x-a)$.

Finally, we wish to establish such an inequality for points $x$ and $y$ which are not in the same component of $\Delta_{F}$. If $x$ and $y$ both belong to $P \cap(c, d)$, then we already have $|F(y)-F(x)| \leqslant(M+2)|y-x|$. This leaves three other cases which may occur; it is sufficient for us to indicate the inequality for $x<y, x \in \Delta_{F}$ and $y \in P \cap(c, d)$. Let $(a, b)$ be that component of $\Delta_{F}$ to which $x$ belongs. Then we have

$$
|F(x)-F(b)| \leqslant 5(M+2)(b-x) \text { and }|F(y)-F(b)| \leqslant(M+2)(y-b)
$$

from the above, so $|F(y)-F(x)| \leqslant 5(M+2)(y-x)$.

These inequalities for the difference quotient $|[F(y)-F(x)] /(y-x)|$ thus guarantee that the conditions of [30, Lemma 3.1] can be fulfilled at any point of $P \cap(c, d)$. It follows then that $F$ must be differentiable at each such point, which contradicts the definition of $P$ and proves the theorem.

There are many consequences that can be drawn from Theorem 8.1. 
8.2 Corollary. Under the hypotheses of Theorem 8.1, an exact derivative $F_{E}^{\prime}$ has the Darboux property on the set of differentiability of $F$.

8.3 Corollary. Under the hypotheses of Theorem 8.1, if $F$ vanishes on a dense set, then $F$ is identically zero. Consequently, any two such E-differentiable functions which agree on a dense set must agree everywhere.

Some final corollaries shed some light on a large number of monotonicity theorems which have appeared in the literature in recent years. With the help of Theorem 8.1, we can easily establish a reduction theorem which indicates that monotonicity theorems which hold for differentiable functions must also hold for $E$-differentiable functions under appropriate assumptions on the paths $\left\{E_{x}: x \in R\right\}$. This generalizes a theorem on approximate derivatives given in [30]. To formulate this precisely we introduce some notation. Let $\Delta$ denote the family of differentiable functions, $\Delta_{E}$ the family of $E$-differentiable functions whose derivatives are Baire 1, where $E=\left\{E_{x}: x \in R\right\}$ is a system of paths, and let $\mathcal{N}$ denote the family of all nondecreasing functions. For any family $\mathcal{S}$ of functions on $R$ and any interval $I \subset R$, we write $S(I)$ as the family of restrictions of functions in $\mathcal{S}$ to $I$.

8.4 THEOREM. Let $E=\left\{E_{x}: x \in R\right\}$ be a system of paths that are nonporous and satisfy the intersection condition. Let $\mathcal{P}$ be a family of functions on $R$. If $\Delta(I) \cap \mathcal{P}(I)$ $\subset \Re(I)$ for every interval $I \subset R$, then $\Delta_{E}(I) \cap \mathscr{P}(I) \subset \Re(I)$ for all $I \subset R$.

Proof. If $F$ is in $\Delta_{E}(I)$ but not in $\Re(I)$ for some interval $I$, then there must be a point $x_{0} \in I$ at which $F_{E}^{\prime}\left(x_{0}\right)<0$, otherwise, by Theorem 4.7, $F$ would have to be nondecreasing on $I$. By Theorem 8.1, there must be an interval $J \subset I$ on which $F$ is differentiable and $F^{\prime}$ assumes some negative values. But then $F$ cannot be in $\mathcal{P}$ since that implies $\Delta(J) \cap \mathscr{P}(J)$ is not contained in $\Re(J)$. This completes the proof.

By choosing various families $\mathcal{P}$ we obtain a number of monotonicity theorems as corollaries. For the first of these, we merely take $\mathcal{P}$ as the family of all functions whose $E$-derivatives are nonnegative a.e. on the set where $F$ is differentiable.

8.5 COROLlary. Let $E=\left\{E_{x}: x \in R\right\}$ be a system of paths that are nonporous and satisfy the intersection condition. If $F$ is E-differentiable, $F_{E}^{\prime}$ is Baire 1 , and $F_{E}^{\prime}(x) \geqslant 0$ for almost every $x$ at which $F$ is differentiable, then $F$ is nondecreasing.

Similarly, we obtain in the obvious way

8.6 Corollary. Let $E=\left\{E_{x}: x \in R\right\}$ be a system of paths that are nonporous and satisfy the intersection condition. Then, if $F$ is E-differentiable, $F_{E}^{\prime}$ is Baire 1 , and $F$ is nondecreasing on every interval on which $F$ is also differentiable, $F$ is nondecreasing.

Note that a feature of this theorem and its corollaries is that the $E$-differentiable function that is proved to be nondecreasing must, in fact, because of Theorem 7.5, be differentiable. The negative of the Cantor function provides a ready counterexample to a number of plausible, but false, variants of these corollaries. 


\section{REFERENCES}

1. S. Agronsky, R. Biskner, A. Bruckner and J. Mařik, Representations of functions by derivatices. Trans. Amer. Math. Soc. 263 (1981), 493-500.

2. C. L. Belna, M. J. Evans and P. D. Humke, Symmetric and ordinary differentiation. Proc. Amer. Math. Soc. 72 (1978), 261-267.

3. A. M. Bruckner, Some observations about Denjoy's preponderant derivative, Bull. Math. Soc. Sci. Math. R. S. Roumaine (N.S.) 21 (69) (1977), 2-10.

4. _ Differentiation of real functions, Lecture Notes in Math., vol. 659, Springer, Berlin and New York, 1978.

5. __ On the differentiation of integrals in euclidean spaces, Fund. Math. 66 (1969), 129-135.

6. A. Bruckner and C. Goffman, The boundary behatiour of real functions in the upper half-plane. Rev. Roumaine Math. Pures Appl. 11 (1966), 507-518.

7. , Approximate differentiation, Real Anal. Exchange 6 (1980/81), 9-65.

8. H. Croft, $A$ note on a Darboux continuous function, J. London Math. Soc. 38 (1963), 9-10.

9. A. Denjoy, Sur les fonctions dérivées sommables, Bull. Soc. Math. France 43 (1915), 161-248.

10. Sur une propriété des fonctions dérivées, Enseignement Math. 18 (1916), 320-328.

11. E. P. Dolženko, Boundary properties of real functions, Math. USSR-Izv. 1 (1967), 1-12.

12. H. W. Ellis, Darboux properties and applications to non-absolutely convergent integrals, Canad. J. Math. 3 (1951), 471-484.

13. M. J. Evans and P. D. Humke, The equality of unilateral derivates, Proc. Amer. Math. Soc. 79 (1980), 609-613.

14. A. Gleyzal, Interval functions, Duke Math. J. 8 (1941), 223-230.

15. C. Goffman and C. Neugebauer, On approximate derivatives, Proc. Amer. Math. Soc. 11 (1960), 962-966.

16. L_ Linear continuous functions, Proc. Amer. Math. Soc. 12 (1961), 997-998.

17. V. Jarnik, Über die Differenzierbarkeit stetizer Funktionen, Fund. Math. 21 (1933), 48-58.

18. A. Khintchine, Recherches sur la structure des fonctions mesurables, Fund. Math. 9 (1927), 212-279.

19. M. Laczkovich, On the Baire class of selective derivatives, Acta. Math. Sci. Hungar. 29 (1977), 99- 105 .

20. S. Marcus, La dérivee approximative qualitative, Com. Acad. R. P. Romaine 3 (1953), 361-364.

21. M. Mastalerz-Wawrznczak, On a certain condition of the monotonicity of functions, Fund. Math. 97 (1977), 187-198.

22. L. Mišik, Über approximative derivierte Zahlen monotoner Funktionen, Czechoslovak Math. J. 26 (101) (1976), 579-583.

23. H. Oliver, The exact Peano derivative, Trans. Amer. Math. Soc. 76 (1954), 444-456.

24. R. J. O’Malley, Baire* 1, Darboux functions, Proc. Amer. Math. Soc. 60 (1976), 187-192.

25. __ Selective derivates, Acta. Math. Acad. Sci. Hungar. 29 (1977), 77-97.

26. Decomposition of approximate derivatives, Proc. Amer. Math. Soc. 69 (1978), 243-247.

27. - Selective derivatives and the $M_{2}$ or Denjoy-Clarkson properties, Acta Math. Acad. Sci.

Hungar. 36 (1980), 195-199.

28. __ Selective differentiation: Redefining selections (submitted).

29. __ Bi-selective derivatives are of honorary Baire class 2 (submitted).

30. R. J. O'Malley and C. E. Weil, The oscillatory behaviour of certain derivatives, Trans. Amer. Math.

Soc. 234 (1977), 467-481.

31. Composite differentiation (in preparation)

32. G. Petruska and M. Laczkovitch, Remarks on a problem of A. M. Bruckner, Acta. Math. Acad. Sci.

Hungar. 38 (1981), 205-214.

33. S. Saks, Theory of the integral Monografie Mat. 7, PWN, Warszawa, 1937.

34. J. Scholz, Essential derivations of functions in $C[a, b]$ (to appear).

35. G. Sindalovski, Derivatives of continuous functions, Izv. Akad. Nauk SSSR 32 (1968). (Russian)

36. T. Swiagtkowski, On a certain generalization of the notation of the derivative (in Polish), Zeszyty

Nauk. Politech. Lódz. Mat. No. 149 (1972), 89-103.

37. G. Tolstoff, Sur la dérivee approximative exacte, Rec. Math. (Mat. Sbornik) N.S. (1938), 499-504.

38. B. S. Thomson, Monotonicity theorems, Real Anal. Exchange 6 (1980/81), 209-234.

39. , Monotonicity theorems, Proc. Amer. Math. Soc. 83 (1981), 547-552.

40. S. Verblunsky, On the Peano derivatives, Proc. London Math. Soc. (3) 22 (1971), 313-324. 
41. C. Weil, On properties of derivatives, Trans. Amer. Math. Soc. 114 (1965), 363-376.

42. , On approximate and Peano derivatives, Proc. Amer. Math. Soc. 20 (1969), 487-490.

43. , A property for certain derivatives, Indiana Univ. Math. J. 23 (1973/74), 527-536.

44. G. C. Young, On the derivates of a function, Proc. London Math. Soc. (2) 15 (1916), 360-384.

45. W. H. Young, Oscillating successions of continuous functions, Proc. London Math. Soc. 6 (1908), $805-807$

46. Z. Zahorski, Sur la première dérivée, Trans. Amer. Math. Soc. 69 (1950), 1-54.

Department of Mathematics, University of California, Santa Barbara, California 93106

Department of Mathematics, University of Wisconsin, Milwaukee, Wisconsin 53201

Department of Mathematics, Simon Fraser University, Burnaby, British Columbia, Canada V5A 1S6 